\title{
Estudio retrospectivo de 11 casos de estesioneuroblastomas tratados en el Hospital Santa Creu i Sant Pau entre los años 2000 y 2008 más revisión de la literatura
}

\author{
F. Muñoz; P. Tresserras; J.R. Montserrat*; F.J. Sancho** y F. Bartumeus \\ Servicios de Neurocirugía, Otorrinolaringología* y Anatomía Patológica**. Hospital Santa Creu i Sant Pau. Barcelona.
}

Resumen

Introducción. El estesioneuroblastoma (ENB) es un tumor maligno de origen neuroectodérmico poco habitual que afecta principalmente a la fosa craneal anterior y cavidad nasal.

Objetivos. Revisar nuestra experiencia en el manejo de los ENB, evaluar la validez del diagnóstico anatomopatológico, el tipo de tratamiento y factores pronósticos de la enfermedad comparando nuestros hallazgos con los descritos en la literatura.

Material y métodos. Estudio retrospectivo de 11 casos de ENB tratados en nuestro hospital en el periodo comprendido entre 2000 y 2008. Se realiza análisis estadístico sobre la existencia de factores pronósticos. Se revisa la bibliografía publicada sobre el ENB entre 1990 y 2009.

Resultados. Se trataron 3 mujeres y 8 hombres, cuya edad media fue 42 años (rango 20-71 años). El síntoma inicial fue la obstrucción nasal $(81 \%)$, epistaxis $(27 \%)$, disminución de agudeza visual $(\mathbf{1 8 \%})$ ), cefalea y otros. Según la clasificación de Kadish, 2 son estadío B y 9 estadío C; según la clasificación de Dulguerov, 2 son T2, 3 son T3 y 6 son T4. El resultado histológico según la clasificación de Hyams fue de 2 casos en grado I, 4 en grado II, 3 en grado III y 2 en grado IV. Dos de los casos inicialmente catalogados en grado IV cambiaron de diagnóstico en la segunda biopsia a tumor indiferenciado. Se realizó un abordaje subcraneal en $73 \%$ (8 casos) con comprobación de la exéresis completa mediante endoscopia endonasal. De ellos, tras radioterapia coadyuvante en todos y en uno quimioterapia, el $62 \%$ (5 pacientes) están vivos sin enfermedad, $12,5 \%$ (1) vivo con enfermedad controlada, $25 \%$ (2) muerte por progresión de la enfermedad. En 1 paciente se realizó resección subtotal por endoscopia endonasal más radioterapia y está vivo sin enfermedad. En los otros 2 casos, que se consideraron irresecables, se realizó biopsia endonasal más quimioterapia y radioterapia;

Recibido: 4-03-10. Aceptado: 28-05-10 uno está vivo con enfermedad controlada y el otro fue éxitus por progresión de la enfermedad. Se realizó RT coadyuvante en todos los casos y QT adyuvante en 5 de los pacientes. El grado histológico de Hyams se ha encontrado como factor pronóstico estadísticamente significativo en la predicción de menor periodo libre de enfermedad en nuestra serie.

Conclusiones. En el diagnóstico histopatológico del ENB, la gradación de Hyams puede ser válida considerando los grado IV como la variante más avanzada siendo en ocasiones difícil de diferenciar de otros tumores indiferenciados.

El abordaje subcraneal o resección craneofacial en estadíos avanzados (Kadish C y algunos B) creemos que sigue siendo de elección como primer tratamiento. La radioterapia es coadyuvante en todos los casos y la quimioterapia en casos seleccionados.

El único factor pronóstico que hemos podido extraer de nuestra serie es el grado histológico según la clasificación de Hyams.

PALABRAS CLAVE: Estesioneuroblastoma olfatorio. Tratamiento quirúrgico. Resultado. Supervivencia. Revisión de la literatura.

A retrospective study about 11 cases of Esthesioneuroblastomas treated in Hospital Santa Creu i Sant Pau between 2000 and 2008 and literature review

\section{Summary}

Introduction. Esthesioneuroblastoma (ENB) is a very uncommon malignant tumor with a neuroectodermal origin that usually involves the anterior cranial fossa and nasal cavity.

Objectives. To review our experience in the management of ENB and assess the validity of the histopathological diagnosis, modality of treatment and prognostic factors of the disease comparing our findings with the literature.

Methods. A retrospective study of 11 cases with the 
diagnosis of esthesioneuroblastoma treated in our hospital between 2000 and 2008. Statistical analysis was performed in search for prognostic factors. The bibliography about ENB published between 1990 and 2009 was reviewed

Results. There were 3 women and 8 men, with a mean age of 42 years old (range 20-71y). Their symptoms upon admission were nasal obstruction (81\%), epistaxis $(27 \%)$, visual loss $(18 \%)$, headache and others. According to the Kadish Stage, 2 were stage $B$ and 9 were stage C. Dulguerov and Calcaterra Classification was also used: 2 were T2, 3 were T3 and 6 were T4. The hystopathological result according to the Hyams classification was: 2 cases in stage $I, 4$ in stage $I$, 3 in stage III and 2 in stage IV. The two cases classified in stage IV changed the diagnosis to undifferenciate tumor in the second biopsy. A subcranial approach was performed in 8 cases combined with endonasal endoscopy to confirm the total removal, followed by radiotherapy in all and chemotherapy in one case, resulting on $62 \%(5$ patients) being alive without disease, $12,5 \%$ (1 p) alive with disease, and 25\% (2 p) dead of disease. Another patient was operated by a single endonasal endoscopic approach and a subtotal removal was achieved. This patient is alive without disease. The other 2 patients were treated by biopsy plus radiotherapy and chemotherapy, because they were considered unresectable, and one of them is alive with disease and the other one is dead of disease. Radiotherapy was performed in all cases and chemotherapy in 5 cases. The hystopathological grading system of Hyams was considered statistically significant as a prognostic factor of disease-free survival.

Conclusions. When the hystopathological diagnosis of ENB is considered, the Hyams classification can be valid considering grade IV as an advanced stage that is sometimes difficult to differentiate from other undiferentiated tumors.

The subcranial approach or craneofacial resection in advanced stages (Kadish $\mathrm{C}$ and some $\mathrm{B}$ ) should be considered as the first treatment of choice. Radiotherapy is indicated in all cases and chemotherapy in selected cases. Hyams' classification was the only staging system that proved useful as a prognostic factor in our series.

KEY WORDS: Olfactory esthesioneuroblastoma. Surgery. Treatment outcome. Survival. Review.

\section{Introducción}

El estesioneuroblastoma (ENB) es una lesión neuroepitelial maligna que proviene del epitelio olfatorio de la cavidad nasal y constituye el 3\% de los tumores endonasales. Este tumor crece a través de la lámina cribosa, el tercio superior del septo nasal y de la parte superior del cornete superior. Fue descrito inicialmente por Berger y Richard $^{3}$ en 1924, quienes le dieron el nombre de esthésioneuroépithéliome olfactif. El origen celular de este tumor no está completamente definido, por lo que ha recibido varios nombres, siendo dos de ellos los más utilizados recientemente: estesioneuroblastoma y neuroblastoma olfatorio. Aproximadamente 950 casos aparecieron en la literatura desde su descripción en 1924 hasta $1997^{7}$ y en un último censo del 2005, son aproximadamente 1.500 casos publicados ${ }^{24}$.

Esta neoplasia no tiene predominancia por ninguno de los dos sexos. Afecta a un rango de edad amplio, que va desde los 2 a los 90 años, aunque presenta un pico bimodal en la segunda década y después en la sexta década de la vida. No obstante, la mayor incidencia se sitúa alrededor de los 50 años.

El hecho de que el ENB sea un tumor muy poco frecuente y que los síntomas pueden asemejar a lesiones de tipo benigno mucho más habituales, hace que su diagnóstico sea difícil. La media de retraso entre inicio de los síntomas y el diagnóstico es de 6 meses. Los síntomas principales son la obstrucción nasal, anosmia, epistaxis, dolor en la zona nasal, lagrimeo excesivo y pérdida de visión. La presentación más habitual es un paciente que refiere obstrucción nasal unilateral de meses o años de evolución.

Las pruebas radiológicas recomendadas para completar el diagnóstico serían la tomografía computarizada (TC), donde veríamos una lesión hipercaptante situada en la parte superior de la cavidad nasal que produce una erosión sobre las estructuras óseas adyacentes, y una resonancia magnética $(\mathrm{RM})$, donde se podría observar con más detalle la extensión intracraneal y afectación de partes blandas.

El patrón histológico está formado básicamente por una población relativamente homogénea de pequeñas células redondas indiferenciadas, establecidas en conglomerados celulares dentro de un estroma fibrilar variable. Entra dentro del diagnóstico diferencial de tumores con células redondas de la región de cabeza y cuello. La presencia de un estroma intercelular fibrilar junto con la presencia de rosetas de Homer-Wright en una neoplasia de la parte superior de la cavidad nasal es considerada como diagnóstico de ENB. Dentro de las diferentes clasificaciones histológicas que se han publicado la más utilizada es la gradación de Hyams y Michaels $^{26}$ (ver tabla 1). Básicamente se establecen dos grados (I y II) que son considerados bien diferenciados y dos grado (III y IV) considerados mal diferenciados. Existe una importante similitud histológica entre los grados más altos (III y IV) con otras neoplasias pobremente diferenciadas como el carcinoma indiferenciado, incluso algunos autores no llegan a reconocer la existencia del grado IV catalogándolo dentro de los carcinomas indiferenciados ${ }^{23,35}$. 
Tabla 1

Clasificación histopatológica de Hyams

\begin{tabular}{|c|c|l|l|l|l|l|}
\hline Grado & $\begin{array}{l}\text { Preservación } \\
\text { arquitectura } \\
\text { lobular }\end{array}$ & $\begin{array}{l}\text { Índice } \\
\text { mitótico }\end{array}$ & $\begin{array}{l}\text { Polimorfismo } \\
\text { nuclear }\end{array}$ & $\begin{array}{l}\text { Matriz } \\
\text { fibrilar }\end{array}$ & Rosetas & Necrosis \\
\hline I & + & ninguno & ninguno & prominente & HW & no \\
\hline II & + & bajo & moderado & presente & HW & no \\
\hline III & $+/-$ & moderado & prominente & baja & FW & rara \\
\hline IV & $+/-$ & alto & marcado & ausente & rara & frecuente \\
\hline
\end{tabular}

HW: Homer Wright; FW: Flexner-Wintersteiner

El origen exacto del ENB es una incógnita. Algunos hallazgos han sugerido que puede proceder del órgano vomeronasal de Jacobson, del ganglio esfenopalatino, de la placoda olfatoria ectodérmica, del ganglio de Loci y de los ganglios autonómicos de la mucosa nasal. No obstante, los estudios más actuales relacionan el ENB con las células progenitoras basales del epitelio olfatorio ${ }^{7}$.

Se ha propuesto también la inclusión del ENB dentro de la familia de los sarcomas de Ewing o de los tumores neuroectodérmicos primitivos (PNET), por la identificación en algunos casos de la translocación (11:22), que es considerada específica del sarcoma de Ewing ${ }^{56}$. Estudios con fluorescencia de hibridación in situ y PCR, no han confirmado esta translocación en el $\mathrm{ENB}^{18}$.

No ha sido documentado claramente un agente etiológico o de exposición para el ENB en humanos. En estudio con roedores sí que se podía inducir la aparición de este tipo de tumor con la exposición de compuestos de nitrosamina. En gatos con ENB espontáneos y en ratas transgénicas que desarrollaban ENB, han sido demostradas la aparición de partículas retrovirales tipo $\mathrm{C}^{54}$. El papel de secuencias retrovirales en humanos no queda esclarecido.

El comportamiento biológico del neuroblastoma olfatorio es muy variable. El rango va desde un crecimiento indolente, con pacientes que han sobrevivido más de 20 años, a neoplasias muy agresivas, con supervivencias de pocos meses. El crecimiento se produce por invasión local, destrucción progresiva de la lámina cribosa, invasión de fosas nasales y senos paranasales, base del cráneo, rinofaringe, fosa craneal anterior, meninges y lóbulos frontales. La incidencia de metástasis es aproximadamente de un 10 al 33\% en el momento del diagnóstico. El lugar más común de metástasis son los ganglios de la región cervical, que ocurre entre un 10 al 33\% de los pacientes ${ }^{7}$. Metástasis a distancia se encuentran entre un 12 al 25\% de los pacientes, siendo el pulmón, el cerebro y el hueso los lugares más comunes. De todas formas la mayoría de estudios que examinan el comportamiento de esta neoplasia son revisiones retrospectivas debido a la rareza del mismo.

Kadish y colaboradores $^{29}$, en 1976, fueron los primeros en proponer una clasificación para el estadiaje del tumor. Establecieron 3 categorías: grupo A, tumor limitado a fosa nasal; grupo B, tumor que se extiende a los senos paranasales; grupo $\mathrm{C}$, extensión que sobrepasa los senos paranasales u ocasiona una metástasis (ver tabla 2). Posteriormente, en al año 1992, Dulguerov y Calcaterra ${ }^{18}$ propusieron una nueva clasificación más descriptiva, basándose en el sistema TNM: T1: tumor que afecta a fosas nasales y/o senos paranasales (excluyendo el seno esfenoidal); $\mathrm{T} 2$, tumor que afecta a fosas nasales y senos paranasales (incluyendo el seno esfenoidal), con infiltración o erosión de la lámina cribosa; T3, tumor que se extiende a órbita o a fosa craneal anterior sin invasión de la duramadre; T4, invasión meníngea o cerebral. El resto de clasificación no varía respecto al sistema clásico TNM: N0-1, según si existe invasión ganglionar y M0-1, según si hay metástasis a distancia. Las dos clasificaciones se pueden aplicar antes del tratamiento y están basadas en las pruebas radiológicas de TC y RM. Su aplicación tiene importancia a la hora de decidir qué tipo de tratamiento se debe realizar así como establecer un pronóstico (ver tabla 3).

Tabla 2

Clasificación de Kadish

\begin{tabular}{|c|c|}
\hline Estadío & Descripción \\
\hline A & tumor limitado a fosa nasal \\
\hline B & tumor que se extiende a los senos paranasales \\
\hline $\mathrm{C}$ & $\begin{array}{l}\text { extensión que sobrepasa los senos paranasales } \\
\text { u ocasiona una metástasis }\end{array}$ \\
\hline
\end{tabular}


Tabla 3

Clasificación de Dulguerov y Calcaterra

\begin{tabular}{|l|l|}
\hline Estadío & \multicolumn{1}{c|}{ Descripción } \\
\hline T1 & tumor que afecta a fosas nasales y/o senos paranasales (excluyendo el seno esfenoidal) \\
\hline T2 & $\begin{array}{l}\text { tumor que afecta a fosas nasales y senos paranasales (incluyendo el seno esfenoidal), con infiltración o } \\
\text { erosión de la lámina cribosa }\end{array}$ \\
\hline T3 & tumor que se extiende a órbita o a fosa craneal anterior sin invasión de la duramadre \\
\hline T4 & invasión meníngea o cerebral \\
\hline N0 & No invasión ganglios linfáticos cervicales \\
\hline N1 & Invasión ganglios linfáticos cervicales \\
\hline M0 & No metástasis \\
\hline M1 & Metástasis a distancia \\
\hline
\end{tabular}

El tratamiento del neuroblastoma olfatorio incluye la cirugía, radioterapia, quimioterapia o una combinación de las mismas. La modalidad de tratamiento óptima para este tumor continúa siendo objeto de debate debido al número limitado de pacientes incluidos en las cohortes que aparecen en los artículos. La mayoría coinciden en que la cirugía es el tratamiento de elección ${ }^{7,14,18,24}$. Aunque el abordaje transfacial fue utilizado en el pasado ${ }^{48}$, los estudios demuestran que la resección craneofacial ofrece una exéresis tumoral en bloque, con baja morbilidad asociada, logra un control local superior y mejora la supervivencia global $^{18,24}$. En los últimos años, parece que el abordaje craneofacial combinando, craneotomía abierta y resección endonasal guiada por endoscopio o vía endonasal únicamente consiguen resecciones muy satisfactorias ${ }^{14}$. La mayoría de autores coinciden en realizar radioterapia coadyuvante postcirugía ${ }^{18,24}$. La quimioterapia, excepto en algunos casos seleccionados en que se administra preoperatoriamente, quedaría relegada para los pacientes considerados inoperables o con múltiples metástasis, combinada con radioterapia ${ }^{4,41}$. A pesar de adoptar estas terapias agresivas, la recurrencia local y regional y las metástasis a distancia son relativamente frecuentes (aproximadamente $29 \%, 16 \%$ y $17 \%$, respectivamente), y en ocasiones pueden aparecer después de largos periodos de seguimiento ${ }^{46}$.

Los factores pronósticos propuestos han sido los estadiajes de Kadish y Dulguerov, la clasificación histopatológica de Hyams, la existencia de infiltración tumoral de los ganglios linfáticos o la presencia de metástasis entre $\operatorname{otros}^{7,18,28,38,41,46}$.

Considerando el metaanálisis realizado por Dulguerov ${ }^{18}$ en el 2001, la supervivencia media a los 5 años de los artículos estudiados fue del $45 \%$ y el periodo libre de enferme- dad a los 5 años es del $41 \%$. Sólo en unos pocos estudios se pudo considerar la supervivencia a los 10 años que fue del $52 \%$. Revisando artículos con un número de casos superior a 25 incluyendo estudios más recientes, el rango de supervivencia a los 5 años sería del 55 al $89 \%$ y un periodo libre de enfermedad a los 5 años del 36,5 al 76\% $0^{2,16-17,37,41,60}$.

\section{Material y métodos}

Las historias clínicas de todos los pacientes tratados de ENB en el Hospital Santa Creu i Sant Pau en el periodo comprendido entre el año 2000 y 2008 fueron revisadas retrospectivamente. La razón por la que se decidió analizar los resultados en este periodo de tiempo fue porque se consideró representativa del tipo de tratamiento actual utilizado. Dos de los casos revisados fueron recidivas de tumores diagnosticados y tratados previamente, uno en otro centro y el otro en nuestro hospital, y que fueron tratados de nuevo entre el año 2000 y 2008. Estos dos casos serán excluidos del análisis de supervivencia para evitar un sesgo de selección.

En un caso tratado inicialmente en nuestro hospital, en un segundo tiempo fue tratado en otro centro, pero la recogida de datos ha sido satisfactoria y creemos conveniente incluirlo en el estudio sobretodo porque aportará información relevante para la discusión.

Todas las muestras fueron revisadas por el mismo patólogo (Dr. F.J.S.). Los tumores se clasificaron siguiendo la gradación propuesta por Hyams y Michaels.

Las historias clínicas fueron revisadas analizando los datos demográficos, grado histológico, extensión tumoral, modalidad de tratamiento, complicaciones, recurrencia y supervivencia. Para evaluar el grado de extensión tumoral 


\section{Tabla 4}

Datos demográficos, estadío, grado histológico y síntoma presentación

\begin{tabular}{|c|c|c|c|c|c|c|}
\hline $\begin{array}{l}\mathrm{N}^{\circ} \\
\text { Pac. }\end{array}$ & Edad & $\begin{array}{l}\text { Sexo } \\
\operatorname{Dx}(\mathrm{a})\end{array}$ & Kadish & Dulguerov & Hyams & Síntoma presentación \\
\hline 1 & 57 & V & $\mathrm{C}$ & $\mathrm{T} 4$ & III & Obstrucción nasal, epistaxis \\
\hline 2 & 34 & V & $\mathrm{C}$ & $\mathrm{T} 3$ & II & Epistaxis \\
\hline 3 & 27 & $\mathrm{~V}$ & $\mathrm{~B}$ & $\mathrm{~T} 2$ & I & Obstrucción nasal \\
\hline 4 & 26 & M & $\mathrm{C}$ & $\mathrm{T} 3$ & II & Obstrucción nasal, rinorrea \\
\hline 5 & 24 & V & $\mathrm{C}$ & $\mathrm{T} 4$ & I & Obstrucción nasal, rinorrea \\
\hline 6 & 71 & V & $\mathrm{B}$ & $\mathrm{T} 2$ & III & Obstrucción nasal \\
\hline 7 & 45 & V & $\mathrm{C}$ & $\mathrm{T} 4$ & III & Obstrucción nasal, disminución agudeza visual \\
\hline 8 & 21 & V & $\mathrm{C}$ & $\mathrm{T} 4$ & $\operatorname{IV}(*)$ & Obstrucción nasal \\
\hline 9 & 36 & M & $\mathrm{C}$ & $\mathrm{T} 4$ & $\operatorname{IV}(* *)$ & Epistaxis, cefalea, proptosis \\
\hline 10 & 43 & M & $\mathrm{C}$ & $\mathrm{T} 4$ & II & Obstrucción nasal \\
\hline 11 & 50 & $\mathrm{~V}$ & $\mathrm{C}$ & $\mathrm{T} 3$ & II & Obstrucción nasal \\
\hline
\end{tabular}

Pac.: paciente; Dx: diagnóstico; a: años; V: varón; M: mujer. (*): primera biopsia ENB grado IV, segunda biopsia tumor indiferenciado. $(* *)$ : primera biopsia ENB grado IV, segunda biopsia carcinoma indiferenciado.

se utilizaron las clasificaciones de Kadish y la de Dulguerov-Calcaterra. La decisión terapéutica fue sometida a discusión en las sesiones de base de cráneo y oncología que periódicamente se realizan en nuestro hospital, con la participación de neurocirujanos, otorrinolaringólogos, oncólogos, neurorradiólogos y anatomopatólogos.

Los objetivos del análisis estadístico de este estudio fueron las curvas de supervivencia y del periodo libre de enfermedad estimadas según el método de KaplanMeier. Se determinó la significancia estadística del grado histológico como factor pronóstico de supervivencia y de periodo libre de enfermedad utilizando el test de log-rank. El nivel de significación empleado fue el usual 5\% (alfa = 0,05). Para los cálculos estadísticos se utilizó el programa SPSS versión 17.

La revisión bibliográfica se ha llevado a cabo mediante una búsqueda en Medline utilizando las palabras "esthesioneuroblastoma" y "olfactory neuroblastoma" desde 1990 hasta 2009. Se localizaron 790 artículos. Fueron incluidos en la revisión bibliográfica aquellos artículos con revisiones de series con un mínimo de 5 pacientes con ENB, aquellos artículos que realizan un estudio de metaanálisis sobre tratamientos, resultados y supervivencia y aquellas revisiones de la literatura que aportaban datos de interés para la discusión. De los artículos publicados por una misma institución con inclusión de más pacientes con ENB y actualizaciones de seguimiento, sólo se consideró el artículo más reciente. Fueron excluidos aquellos artículos que no trataban directamente sobre el ENB, aquéllos que trataban fundamentalmente de datos radiológicos, o únicamente se centraban en una determinada técnica quirúrgica o tratamiento quimioterápico o radioterápico. Sólo se incluyeron artículos cuyo lenguaje era el español, inglés, francés, alemán e italiano.

\section{Resultados}

\section{Serie Hospital Santa Creu i Sant Pau}

Un total de 11 pacientes con el diagnóstico de ENB fueron tratados en nuestro Hospital durante el periodo comprendido entre 2000 y 2008. Se realizó una búsqueda previa a estas fechas y fueron identificados 16 pacientes entre 1981 y 2008 , pero como se ha comentado se ha limitado la revisión a los últimos 9 años. Sólo se han incluido dos casos diagnosticados previamente al año 2000 y tratados durante el periodo de estudio, dado que consideramos que el tratamiento recibido es representativo de nuestro protocolo terapéutico (fueron tratados entre el 2000 y el 2008).

La serie consta de 3 mujeres y 8 hombres. La mediana de edad al diagnóstico fue de 35 años (rango de 21-71 años). Únicamente 2 de los pacientes presentaban historia de tabaquismo. El síntoma inicial fue obstrucción nasal $(81 \%)$, epistaxis (27\%), disminución de agudeza 
visual $(18 \%)$, cefalea $(9 \%)$, proptosis $(9 \%)$, crecimiento radiológico (9\%), diplopia (9\%).

La extensión del tumor fue evaluada según las clasificaciones de Kadish y Dulguerov-Calcaterra. Según la clasificación de Kadish, no hay estadío A, 2 son estadío B y 9 son estadío C; según la clasificación de Dulguerov-Calcaterra, no hay T1, 2 son T2, 3 son T3 y 6 son T4.

El grado histológico según la clasificación de Hyams es de 2 casos grado I, 4 grado II, 3 en grado III y 2 en grado IV. En los dos casos de grado IV, considerados estadío avanzado de la enfermedad, la primera biopsia fue compatible con grado IV, pero en la segunda biopsia de la siguiente cirugía fue considerada como tumor indiferenciado, en uno de los dos casos, y en el otro, como carcinoma indiferenciado. Esta última muestra fue analizada en otro centro y el resultado fue comunicado telefónicamente (ver tabla 4).

La presencia de metástasis ganglionares se constató en uno de los 11 casos mientras que se halló una metástasis cerebral a distancia en otro caso.

La modalidad de tratamiento utilizado fue la siguiente: resección craneofacial (abordaje subcraneal) en el 73\% (8 casos) con comprobación de la exéresis completa mediante endoscopia transnasal. En 1 paciente se realizó resección subtotal por endoscopia endonasal y en los otros 2 casos, que se consideraron irresecables, se realizó una cirugía endonasal limitada con finalidad esencial de precisar el diagnóstico (biopsia "debulking"). En todos los casos se realizó radioterapia y en 5 casos se realizó quimioterapia (hubo otro caso que se trató con QMT antes del año 2000 en otro centro). (Ver tabla 5).

Tabla 5

Tratamiento, resultado y seguimiento

\begin{tabular}{|c|c|c|c|c|c|c|}
\hline $\mathrm{N}^{\mathrm{o}}$ Pac. & Edad & Tratamiento & $\begin{array}{l}\text { RT } \\
\text { (Gy) }\end{array}$ & QMT & $\begin{array}{l}\text { Resultado } \\
\text { final }\end{array}$ & $\begin{array}{l}\text { Seguimiento } \\
\text { (meses) }\end{array}$ \\
\hline 1 & 57 & $\begin{array}{l}\mathrm{RCF}(\mathrm{x} 2)+\mathrm{RT}+ \\
\mathrm{QMT}\end{array}$ & 57,6 & Cisplatino & VSE & 36 \\
\hline 2 & 34 & $\mathrm{RCF}+\mathrm{RT}$ & 50,4 & No & VSE & 84 \\
\hline 3 & 27 & $\mathrm{RCF}+\mathrm{RT}$ & 55,8 & No & VSE & 48 \\
\hline 4 & 26 & $\mathrm{RCF}+\mathrm{RT}$ & 48,6 & No & VSE & 48 \\
\hline 5 & 24 & $\mathrm{RCF}+\mathrm{RT}$ & 56 & No & VSE & 72 \\
\hline 6 & 71 & $\begin{array}{l}\text { Endoscópico } \\
\text { (parcial) + RT }\end{array}$ & 55,8 & No & VSE & 96 \\
\hline 7 & 45 & $\mathrm{RT}+\mathrm{QMT}$ & & $\begin{array}{l}\text { Cisplatino + } 5 \\
\text { fluorouracilo }\end{array}$ & VCE & 24 \\
\hline 8 & 21 & $\begin{array}{l}1^{\mathrm{o}}: \mathrm{QMT}+\mathrm{RCF} \\
+\mathrm{RT} \\
2^{\mathrm{o}}: \mathrm{RCF}+\mathrm{QMT} \\
+\mathrm{RTEF}\end{array}$ & 52 & $\begin{array}{l}1^{\mathrm{o}}: \text { Cisplatino }+ \\
5 \text { fluorouracilo } \\
2^{\mathrm{o}}: \text { Taxotere }+ \\
\text { Cisplatino }\end{array}$ & MDE & 48 \\
\hline 9 & 36 & $\begin{array}{l}1^{\mathrm{o}}: \mathrm{QMT}+\mathrm{RT} \\
3^{\mathrm{o}}: \text { Endoscópico } \\
\text { (parcial) }\end{array}$ & & $\begin{array}{l}\text { Taxotere, } \\
\text { Cisplatino, } 5 \\
\text { fluorouracilo }\end{array}$ & MDE & 24 \\
\hline 10 & 50 & $\begin{array}{l}\text { RPLN + QMT } \\
(1992) \\
\text { RCF (1992) } \\
\text { RCF + RT (2002) }\end{array}$ & 52 & $\begin{array}{l}\text { Cisplatino y } \\
\text { Teniposido } \\
\text { (1992) }\end{array}$ & VSE & $192(*)$ \\
\hline 11 & 61 & $\begin{array}{l}\mathrm{RCF}+\mathrm{RT}(1983) \\
\mathrm{RCF}+\mathrm{RT}(1990) \\
\mathrm{RCF}(2001)+\mathrm{QMT}\end{array}$ & 60 & $\begin{array}{l}\text { Cisplatino y } 5 \\
\text { fluorouracilo }\end{array}$ & MDE & $264(*)$ \\
\hline
\end{tabular}

RCF: resección craneofacial; RT: radioterapia; QMT: quimioterapia; RPLN: resección paralateronasal; RTEF: radioterapia estereotáxica fraccionada; Gy: Grays; VSE: vivo sin enfermedad; VCE: vivo con enfermedad; MDE: muerto de la enfemedad. (*): se excluye del cálculo de media y mediana de seguimiento y del cálculo de las curvas de seguimiento y periodo libre de enfermedad. 
El protocolo terapéutico se basó fundamentalmente en la extensión tumoral. En los casos donde la extensión tumoral era extensa y afectaba a la base de cráneo, estadíos Kadish C y Dulguerov T3 - T4, se realizó una resección craneofacial o abordaje subcraneal extendido clásico de Raveh, con exéresis de la duramadre y parte de parénquima cerebral si estaba infiltrado. La reconstrucción de la base craneal se realizó utilizando la técnica de 3 capas de fascia lata más periostio para tapizar la base craneal anterior y evitar la fístula de líquido cefalorraquídeo (LCR). En 2 casos, que se consideró como enfermedad muy avanzada, considerada irresecable, se realizó como primer tratamiento quimioterapia más radioterapia sin cirugía.

En los dos casos donde la afectación no era tan extensa, estadíos B de Kadish o T2 de Dulguerov, el caso fue sometido a debate en sesión de base de cráneo, siendo en un caso la resección craneofacial de elección y en el segundo caso, se realizó cirugía endoscópica endonasal con resección subtotal del tumor.

La radioterapia fue adyuvante postcirugía en todos los casos.

La quimioterapia se utilizó en aquellos casos en los que hubo recidiva, presentaban metástasis o en aquéllos en los que se consideró el tumor como irresecable. En dos casos se utilizó quimioterapia preoperatoria, hubo buena respuesta y posteriormente se decidió rescate quirúrgico.

El tiempo medio de seguimiento fue de 53,3 meses. La mediana es de 48 meses. De este cálculo, se excluyen los casos que fueron diagnosticados previamente, en los que sería de 16 y 21 años respectivamente (casos números 10 y $11)$.

Las complicaciones que se produjeron fueron de osteomielitis crónica del colgajo óseo en 2 casos, infección de herida en un caso y fístula de LCR que requirió revisión quirúrgica en otro caso.

Si consideramos únicamente los casos diagnosticados a partir del año 2000 ( 9 casos), se observaron 2 recurrencias de los 7 pacientes que se reintervinieron posteriormente. El primer caso de recidiva (paciente número 1) fue a los 24 meses, presentó una recidiva local más metástasis cerebral. En este caso se trató con QMT, con muy buena respuesta, logrando hacer desaparecer radiológicamente la afectación cerebral para posteriormente someterse a cirugía. En el segundo caso (paciente número 8), se realizó QMT preoperatoria con cisplatino +5 fluorouracilo más cirugía y posteriormente reintervención quirúrgica más RT (ver caso ilustrativo número 1 más adelante).

En otros dos casos considerados inicialmente irresecables, se realizó QMT y RT tras biopsia. En un caso (paciente número 7) la enfermedad se ha mantenido controlada tras los periódicos controles de imagen que se han recogido en los 24 meses de seguimiento. En el segundo caso (paciente número 9), se trató inicialmente con QMT y RT por considerarse irresecable, con buena respuesta ini- cial pero con recidiva a los 20 meses. En aquel momento se realizó cirugía endoscópica en otro centro que fue subtotal, siendo éxitus a los 2 meses.

En los dos casos con mayor tiempo de seguimiento (números 10 y 11 de la tabla 5) se realizó un tratamiento combinado. El primero de ellos (número 10), se realizó en 1992 en otro centro una cirugía vía rinotomía lateral con exéresis subtotal asociando QMT con pobre respuesta, a finales de 1992 en el mismo centro se verificó una resección craneofacial sin asociar RT porque se consideró exéresis completa (todo ello en el otro centro). Posteriormente se derivó a nuestro centro para seguir controles. A los 10 años se objetivó recidiva del ENB catalogado como estadío C de Kadish, con invasión orbitaria, y se realizó resección craneofacial más RT coadyuvante, ya en nuestro hospital. Transcurridos 6 años desde la última maniobra terapéutica, el paciente está vivo sin evidencia de enfermedad. El segundo caso (número 11), que fue diagnosticado en 1983, se realizó resección craneofacial en 3 ocasiones (1983, 1990 y 2001) asociándose RT las dos primeras. Presentó metástasis ganglionares en 1995 y 2003, efectuándose un vaciamiento ganglionar. En 2004, presentó una recidiva con diseminación dural e intracraneal con múltiples nódulos, no se consideró tributaria de cirugía y se realizó quimioterapia paliativa. Fue éxitus al año.

Tras el periodo de seguimiento, 5 pacientes $(45,5 \%)$ están vivos sin enfermedad, 3 pacientes $(27,3 \%)$ vivos con enfermedad controlada y 3 pacientes $(27,3 \%)$ muertos de la enfermedad. Dado que una gran parte de los pacientes no tienen un seguimiento superior a los 5 años, la supervivencia a los 2 años es de un $81,2 \%$, mientras que el periodo libre de enfermedad a los 2 años es de 63,3\% (sólo se incluyen los casos diagnosticados en el periodo 2000-2008; 9 casos).

Si utilizamos el análisis estadístico para estimar la supervivencia según el método de Kaplan-Meier (excluimos los casos de más de 10 años de seguimiento como hemos comentado previamente, para evitar un sesgo de

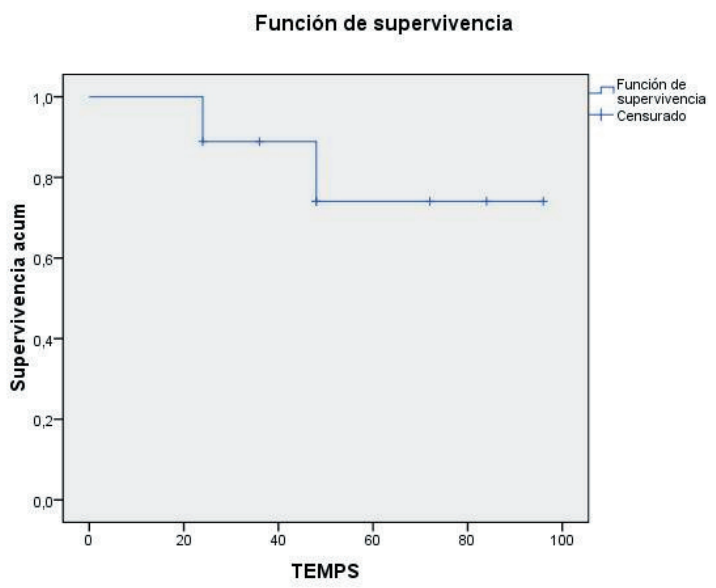

Gráfica 1. Curva de supervivencia 
selección), sería la siguiente gráfica:

En el caso de periodo libre de enfermedad, de los 9 casos comentados, la gráfica sería la siguiente:

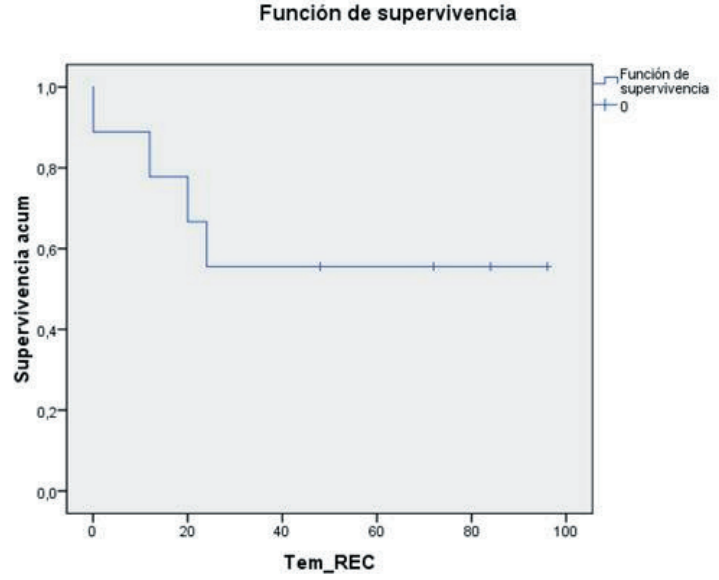

Gráfica 2. Curva de periodo libre de enfermedad. Funciones de supervivencia

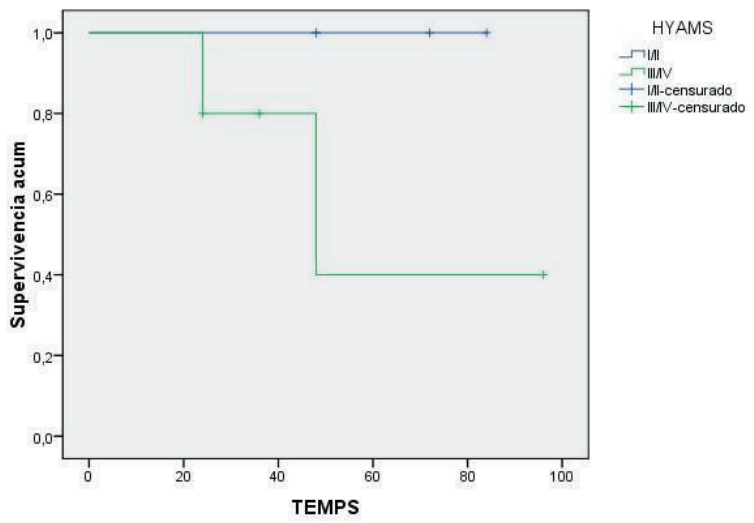

Gráfica 3. Hyams como factor pronóstico de supervivencia.

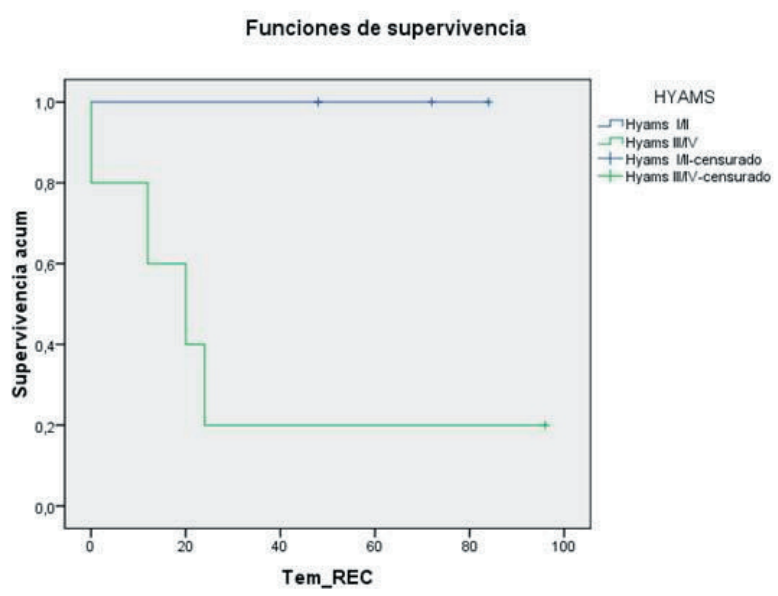

Gráfica 4. Hyams como factor pronóstico de periodo libre de enfermedad.
Se ha realizado un cálculo estadístico para determinar si el grado histológico de Hyams, dividido en grado I/II (baja agresividad) y grado III/IV (alta agresividad) es factor pronóstico de supervivencia y de periodo libre de enfermedad. Sobre la hipótesis de que los grados III/IV sean factores pronósticos de menor supervivencia el resultado no es estadísticamente significativo, $\mathrm{p}=0.105$, pero se observa una tendencia clara a que a mayor agresividad histológica menor supervivencia global (ver gráfica 3)

Sobre si la gradación histológica de Hyams es factor
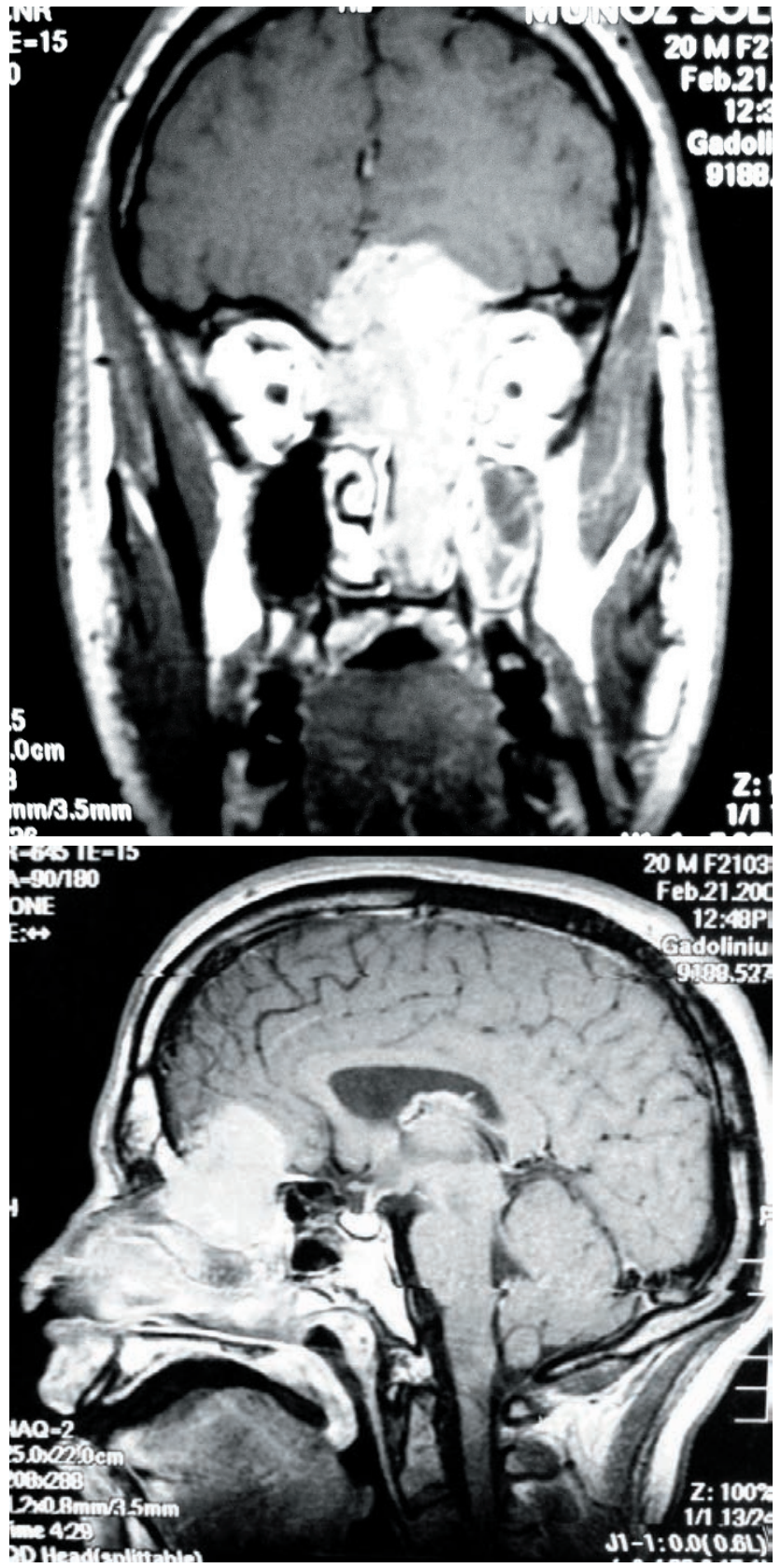

Figuras A y B. RM cerebral pretratamiento. 
pronóstico de periodo libre de enfermedad, en este caso sí que es estadísticamente significativo con una $\mathrm{p}=0,026$, pese a el número tan limitado de pacientes (ver gráfica 4).

\section{Casos ilustrativos}

Caso número 1. Paciente varón de 21 años que a raíz de una historia de obstrucción nasal de meses de evolución se diagnostica por biopsia ENB. En la RM cerebral se observa una gran masa tumoral que invade toda la fosa nasal izquierda seno maxilar, etmoidal y se extiende hacia la base craneal anterior y se hace intracraneal. (ver figuras A y B). El tumor corresponde a una grado C de Kadish y un grado T4 de Dulguerov-Calcaterra.

Dada la extensión tumoral que presentaba el tumor, se decidió realizar QMT preoperatoria con cisplatino y 5fluorouracilo para reducir la masa tumoral. Posteriormente se llevó a cabo una resección craneofacial mediante un abordaje subcraneal extendido consiguiéndose una exéresis macroscópica completa.

El resultado anatomopatológico fue de estesioneuroblastoma grado IV de Hyams. (ver figura C, donde podemos observar la presencia de alguna roseta de FlexnerWintersteiner característica).

Posteriormente se realizó RT (52 Gy). El paciente siguió controles donde no había evidencia de tumor hasta que a los 2 años presentó de nuevo recidiva del tumor (ver figuras D y E).

Se realizó de nuevo resección craneofacial con exéresis estimada del 100\% del tumor. Posteriormente se realizó de nuevo QMT, esta vez, con Taxol y cisplatino y radioterapia estereotáxica fraccionada (54 Gy).

En esta última intervención, el resultado de la biopsia no pudo ser catalogado de ENB sino que fue etiquetado de tumor indiferenciado (ver figura $\mathrm{F}$, donde se ve un tumor indiferenciado con extensas áreas de necrosis).

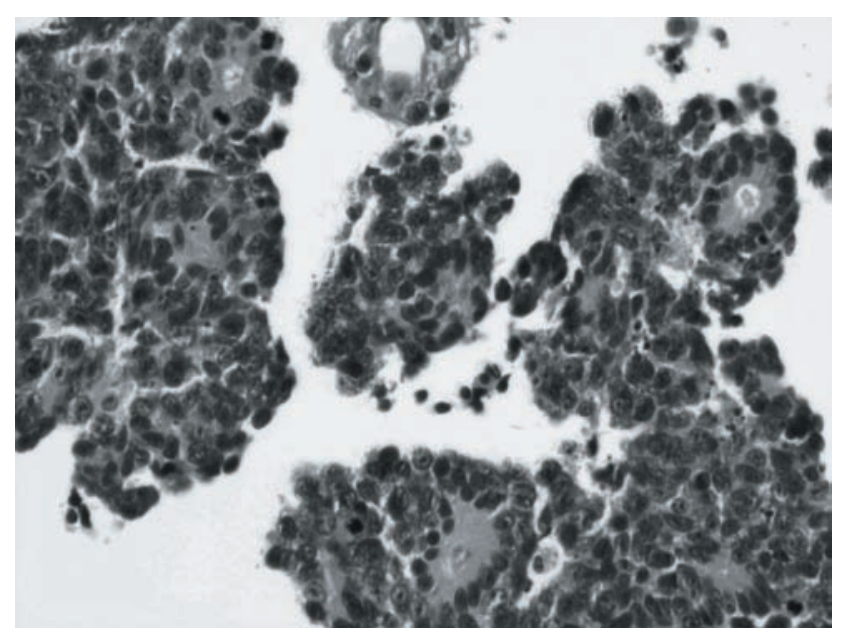

Figura C. Biopsia tras primera cirugía.

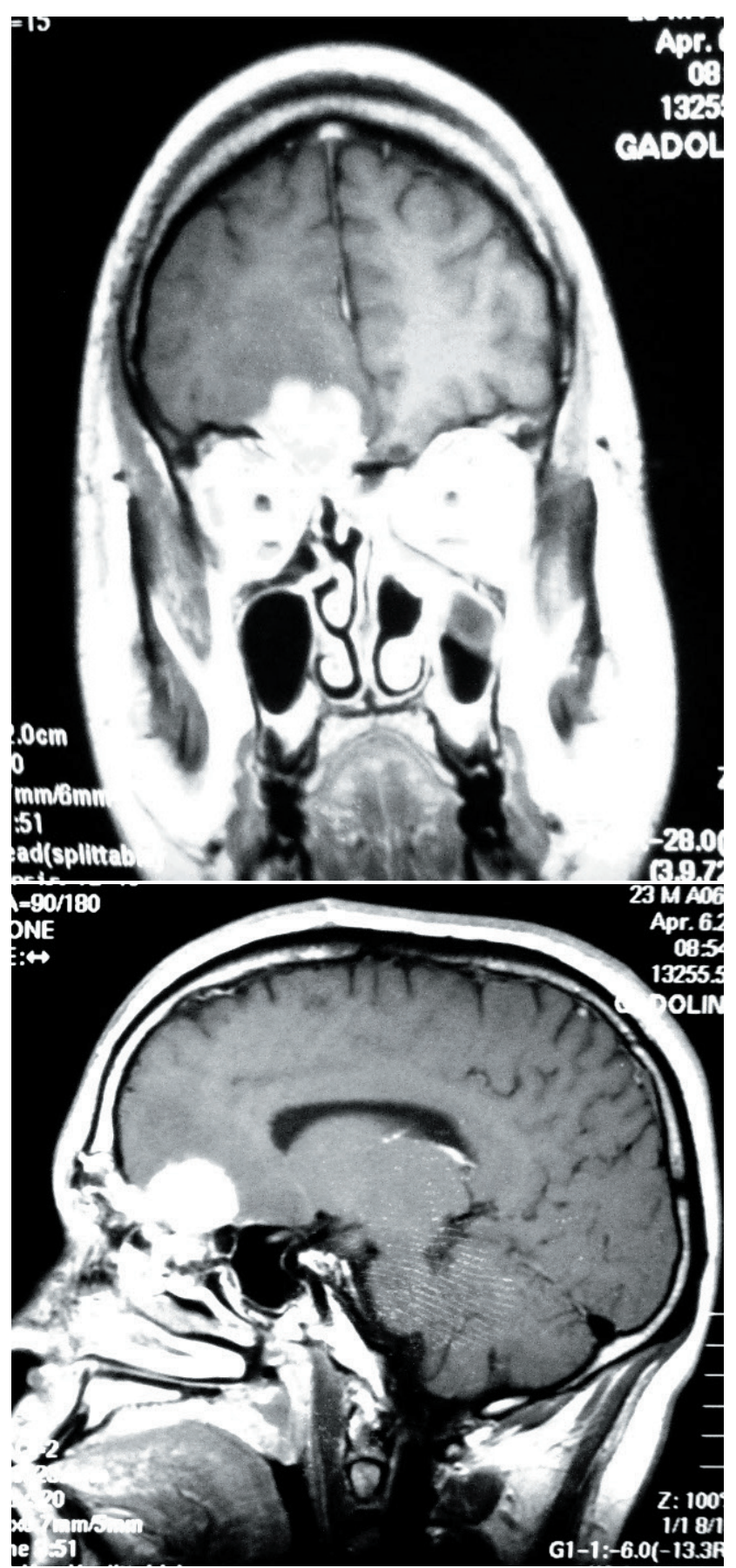

Figuras D y E. Primera recidiva tumoral a los 2 años.

En el control a los 6 meses de la última cirugía no se observó recidiva (ver figuras $\mathrm{G}$ y $\mathrm{H}$ ).

Aproximadamente a los 3 años de realizarse el diagnóstico, el paciente empeoró neurológicamente y en la RM cerebral se observó una extensa recidiva tumoral (ver figuras I y J) que no fue candidata a ningún tratamiento y finalmente fue éxitus al poco tiempo.

Caso número 2. Paciente varón de 27 años con clínica 


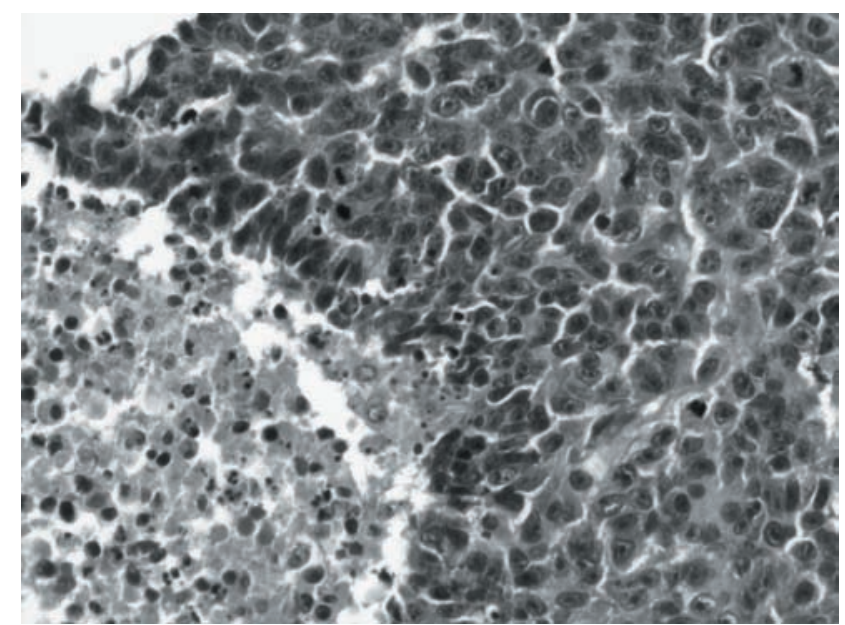

Figura F. Biopsia tras segunda cirugía.

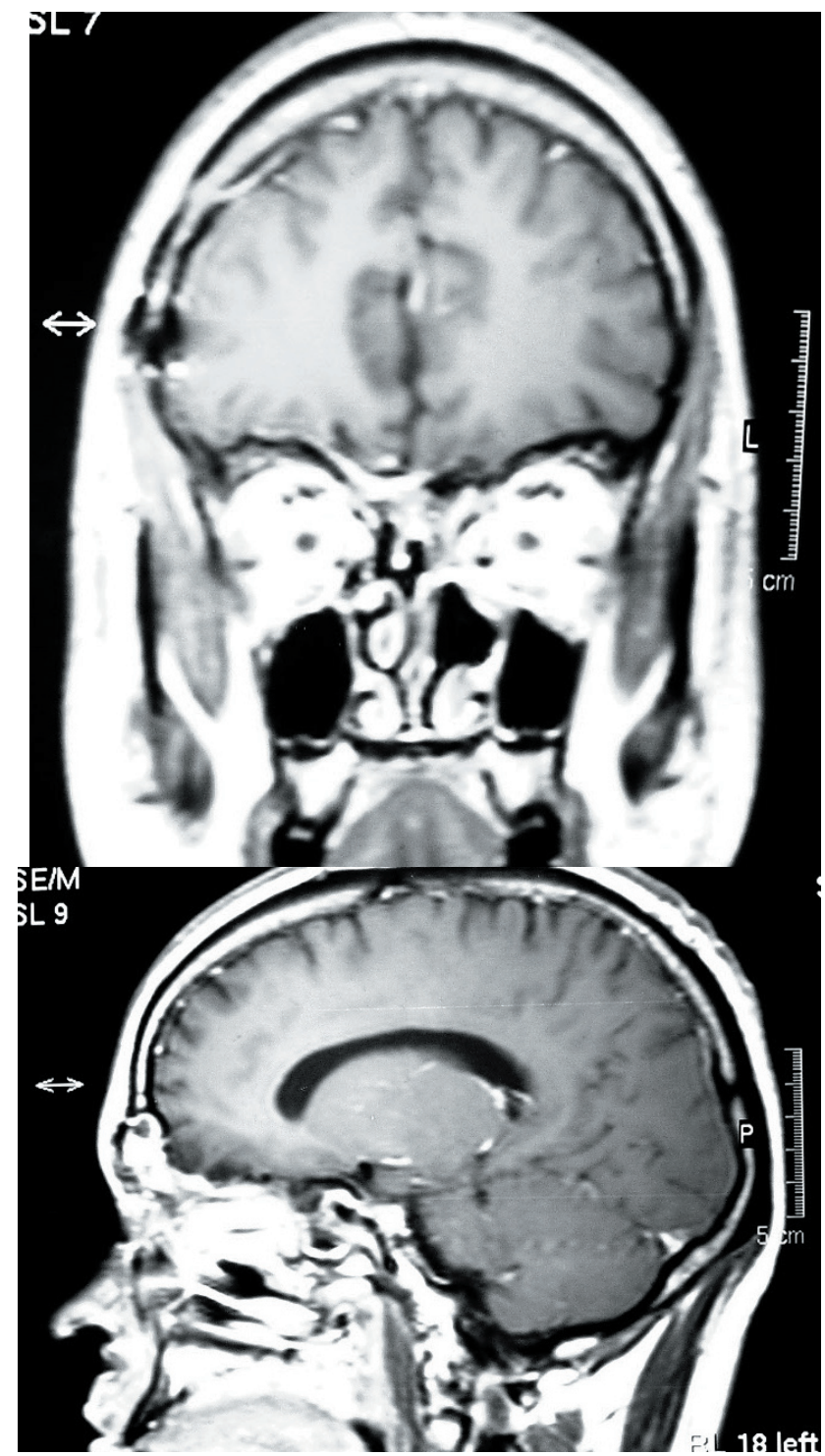

Figuras G y H. Control radiológico a los 6 meses de la segunda cirugía.

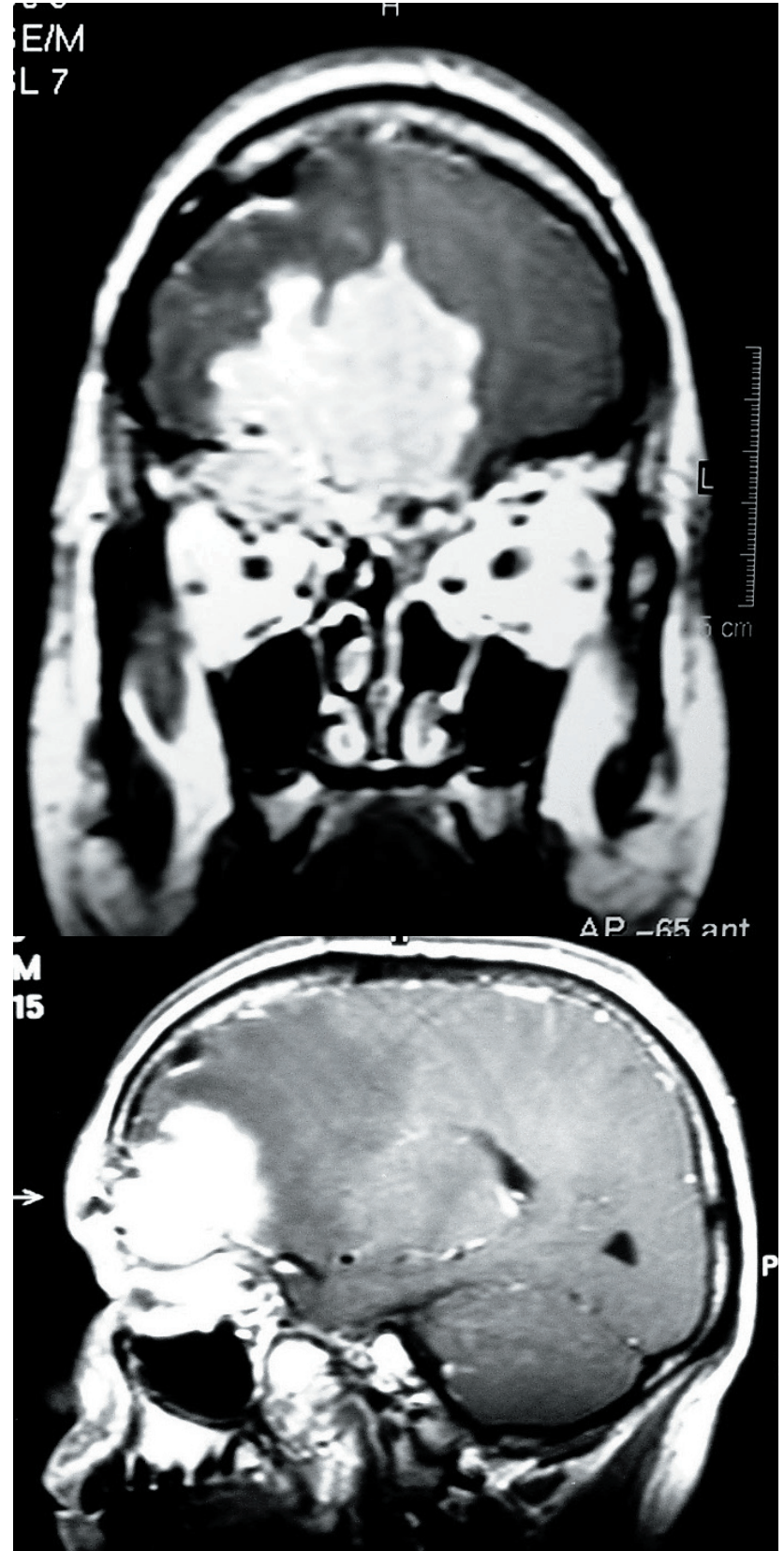

Figuras I y J. Segunda recidiva.

de obstrucción nasal de meses de evolución. Se realiza TC craneal y RM cerebral donde se observa extensa lesión nasosinusal que invade fosa nasal derecha, seno maxilar y celdillas etmoidales (ver figura $\mathrm{K}$ ). Considerado estadío B de Kadish y T2 de Dulguerov-Calcaterra.

El paciente es intervenido mediante resección craneofacial (abordaje subcraneal extendido) con exéresis estimada del $100 \%$. El resultado de anatomía patológica fue de ENB grado I de Hyams. Posteriormente se realizó RT (55,8 Gy). A los 4 años de seguimiento el paciente no presenta evidencia de recidiva (ver figuras L y M). 


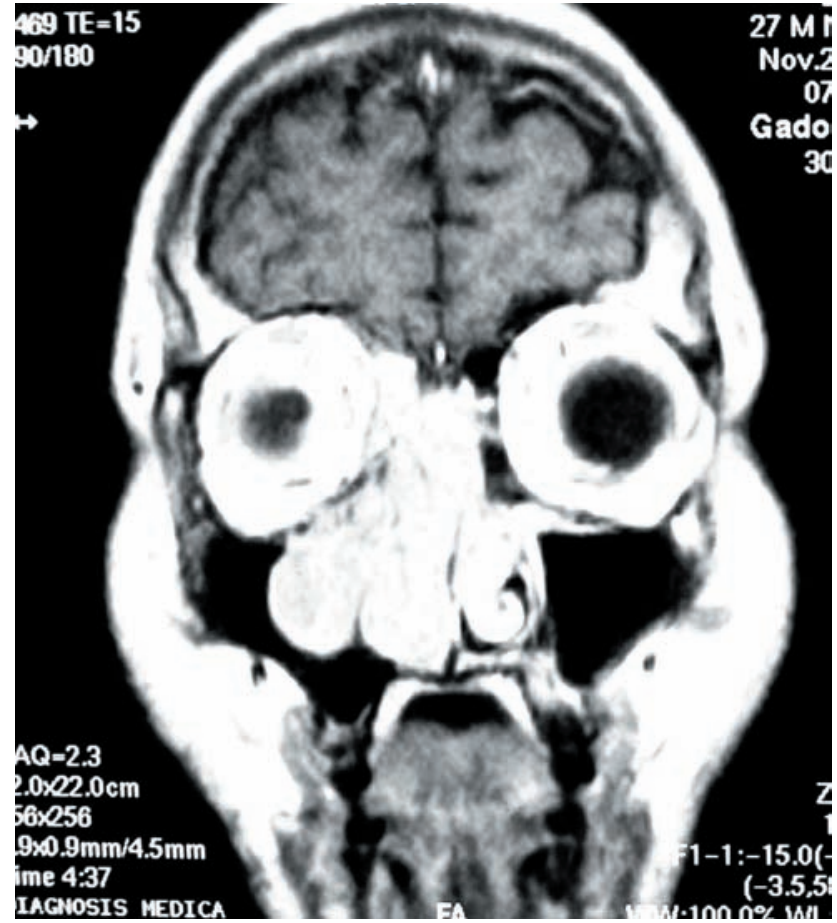

Figura K.

\section{Revisión bibliográfica}

Como se ha comentado previamente de los 790 artículos encontrados, fueron considerados útiles 68 . Se hallaron 45 publicaciones que hablaban sobre estudios retrospectivos de series de más de 5 pacientes. En 2 artículos se realizaban metaanálisis sobre los datos aportados por las diferentes series publicadas hasta el momento ${ }^{14,18}$. En 7 publicaciones se realizaban revisiones sobre el $\mathrm{ENB}^{5-7,24,28,36,49}$. En 4 se hablaban sobre las diferentes técnicas quirúrgicas, sobretodo resección craneofacial y endoscopia ${ }^{15,21-22,60}$. También se incluyeron 5 artículos que trataban sobre datos anatom opatológicos $^{12,23,43,45,52}, 3$ sobre la genética del ENB ${ }^{31,54,56}$, y 3 artículos históricos que fueron considerados interesantes para incluir ${ }^{3,26,29}$

Sobre la controversia que existe sobre el diagnóstico diferencial entre ENB y carcinoma indiferenciado, varios artículos hablan sobre el error diagnóstico que se establece cuando se considera que es un ENB cuando en realidad es un carcinoma indiferenciado. Westra ${ }^{51}$, anatomopatóloga del hospital John Hopkins revisó los espécimenes histológicos de 37 pacientes y encontró que $8(21,6 \%)$ estaban excluidos por no encontrarse los criterios de inclusión diagnósticos de ENB. Cohen ${ }^{12}$ publica una serie de 12 casos diagnosticados inicialmente de ENB, que tras ser revisados sólo en 2 de ellos se consideró que presentaban los criterios diagnósticos de ENB. Otros autores como Levine o Mills, consideran que el grado IV de Hyams no debe ser conside-

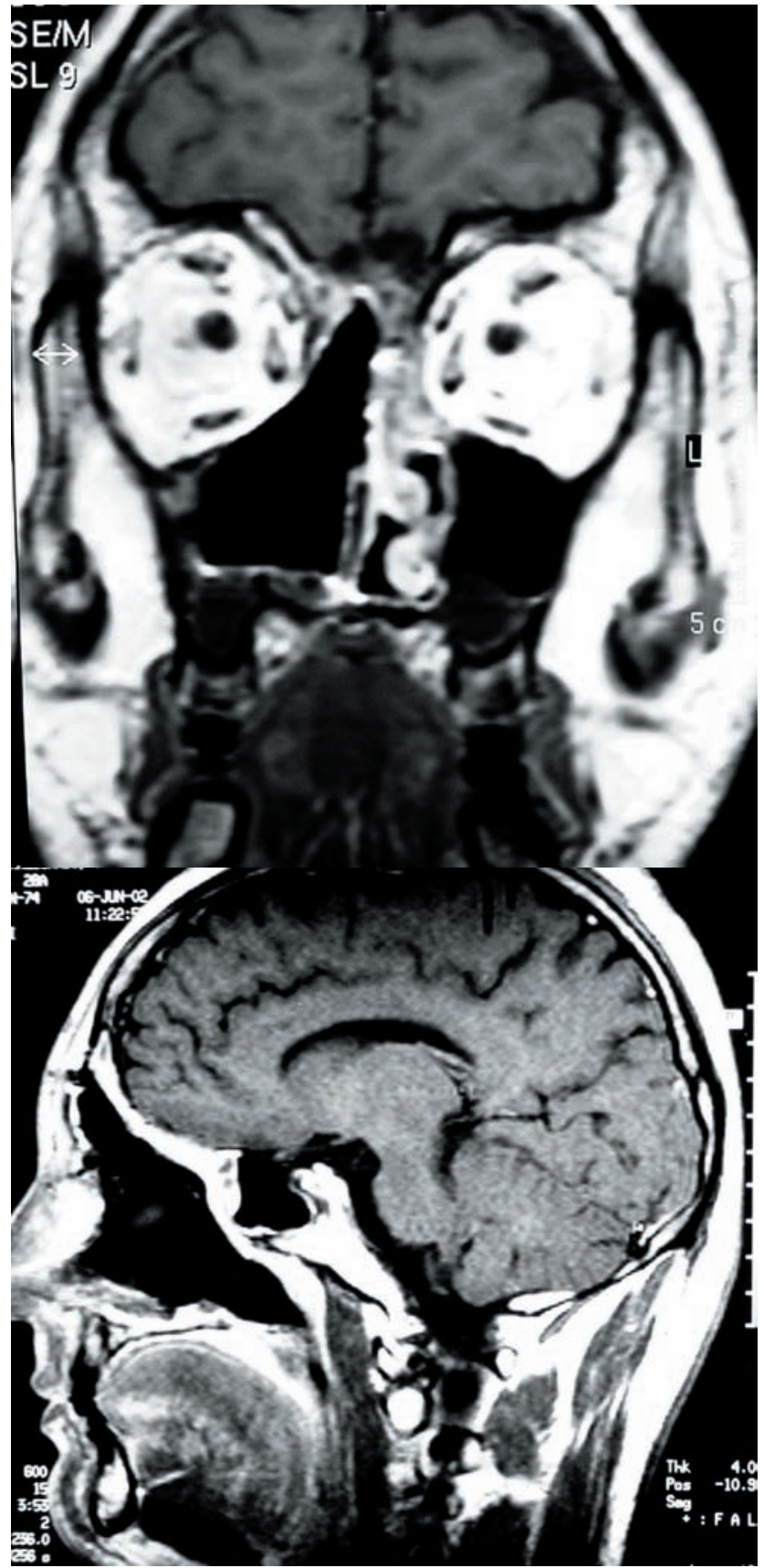

Figuras L y M.

rado como ENB, sino que más bien debería ser clasificado como carcinoma indiferenciado ${ }^{23,35}$.

En el metaanálisis publicado por Dulguerov ${ }^{18}$, que trata sobre 390 pacientes extraídos de 26 publicaciones, observa que las mejores supervivencias se encuentran en el grupo de cirugía más RT (65\%) mientras que cirugía sola es de $48 \%$, cirugía más RT más QMT es del $47 \%$ y $37 \%$ para RT sola. La media de supervivencia global y el periodo libre de enfermedad a los 5 años de los artículos revisados fueron 
Tabla 6

Resultados de algunas series publicadas

\begin{tabular}{|l|l|c|c|c|}
\hline Autor y año publicación & $\mathrm{N}$ & Años estudiados & Supervivencia 5 años & Periodo libre enfermedad 5años \\
\hline Nichols AC (2008) & 10 & $1997-2006$ & $85,7 \%$ & $90 \%$ \\
\hline Bachar G (2008) & 39 & $1972-2006$ & $87,9 \%$ & $76 \%$ \\
\hline McLean (2007) & 21 & & $71,4 \%$ & $59 \%$ \\
\hline Loy (2006) & 50 & $1976-2004$ & - & $86,5 \%$ \\
\hline Diaz (2005) & 30 & $1979-2002$ & $89 \%$ & $69 \%$ \\
\hline Lund (2003) & 41 & $1978-2001$ & $61 \%$ & $77 \%$ \\
\hline Theilgaard (2003) & 40 & $1978-2000$ & $61 \%$ & $50 \%$ \\
\hline Dias (2003) & 35 & $1983-2000$ & $55 \%$ & $46 \%$ \\
\hline
\end{tabular}

de 45\% (desviación estándar (DE): 22) y 41\% (DE: 21) respectivamente. La supervivencia media en los pacientes con grado I-II de Hyams fue del 56\% (DE: 20) comparada con el 25\% (DE:25) en los grado III-IV (odds ratio:6.2). El otro factor pronóstico de supervivencia fue el de la presencia de metástasis ganglionares cervicales (odds ratio: 5.1).

En la revisión realizada por el grupo de Gil-Garcedo ${ }^{24}$, donde incluye 713 casos de la literatura, concluye que la cirugía, sola o combinada es el medio terapéutico más utilizada, siendo la estrategia más habitual la cirugía más RT; la técnica quirúrgica estándar es la resección craneofacial aunque en tumores pequeños (estadíos A) puede ser utilizada el abordaje endoscópico transnasal.

En el metaanálisis realizado por Devaiah ${ }^{14}$, compara los resultados del tratamiento endoscópico con el de cirugía abierta en un periodo de 16 años donde incluyen 361 pacientes. Concluye que los resultados de la cirugía endoscópica son mejores, aunque en la discusión reconoce que los grupos no son comparables dado que en la cirugía abierta existen más casos en estadío avanzado de la enfermedad.

Los resultados del tratamiento de las series más recientes muestran una supervivencia a los 5 años que varía entre el 50 y el $90 \%$ mientras que el periodo libre de enfermedad varía entre el $45-90 \%$. En la siguiente tabla se incluyen los datos de algunas de las series más recientes ${ }^{2,16,17,37,41,42,48,60}$ (tabla 6):

En un estudio realizado por Jethanamest ${ }^{28}$ sobre factores pronósticos, basado en una base de datos sobre 311 pacientes diagnosticados de ENB, observa que la clasificación modificada de Kadish (incluye el estadío D: con metástasis ganglionares cervicales o a distancia), la existencia de metástasis ganglionares, la modalidad de tra- tamiento, la edad del diagnóstico, son factores pronósticos estadísticamente significativos. Estos hallazgos coinciden con los encontrados por otros autores, aunque muchos de ellos también incluyen el grado histológico, la presencia de metástasis a distancia, incluso la afectación orbitaria ${ }^{38}$.

\section{Discusión}

Los datos disponibles en la literatura sobre el ENB, tanto sobre el diagnóstico, tratamiento, factores pronósticos y supervivencia, son la recopilación de numerosas series de pacientes publicadas por la experiencia de una institución en concreto. El número de pacientes por artículo no excede de 50 y los periodos de inclusión de pacientes son demasiado extensos (la mayoría de unos 15-20 años) para dar una idea actualizada sobre el manejo de este tipo de tumores.

El diagnóstico de ENB en nuestros pacientes fue realizado en base a la clasificación de Hyams ${ }^{26}$. En contra de lo que piensan varios autores ${ }^{23,35,52}$, creemos que el grado IV en el ENB sí que existe, dado que es la variante más agresiva y pobremente diferenciada de este tumor, siendo su origen el epitelio olfatorio. Además hemos podido observar, en dos de nuestros pacientes, cómo puede haber una variación en la morfología del ENB tras las sucesivas cirugías que se llevan a cabo, siendo inicialmente considerado un grado IV y luego no llegar a catalogarse de grado IV porque es tan indiferenciado que automáticamente se le adjudica la etiqueta de tumor indiferenciado. Aunque se produzca este hecho, continuamos creyendo que es un ENB dado que inicialmente así lo era. Este concepto de progresión tumoral de un grado IV a tumor indiferenciado, no lo hemos encontrado reflejado en las publicaciones que hemos revisado, 
pero creemos que se debería tener en cuenta. Es más, en línea de lo que estamos hablando, algunos tumores indiferenciados nasosinusales (muchos de ellos carcinomas) podrían haber sido en su origen ENB, por lo que cabe la posibilidad de que sea una patología infradiagnosticada.

No obstante, como se ha comentado previamente, el diagnóstico de ENB en los casos más agresivos, que suelen ser los más indiferenciados, crea una controversia sobre los criterios diagnósticos que se utilizan en cada centro. Los límites de diferenciación son imprecisos y habría que determinar qué mínimo de diferenciación exige cada autor para aceptar que un tumor poco diferenciado se considere un ENB.

Si nos centramos sobre el tratamiento realizado, en 8 de los 11 de nuestros pacientes $(72,7 \%)$ se realizó resección craneofacial seguida de RT como primera opción. Los casos eran estadío B y C de Kadish, por lo que la elección del tipo de tratamiento está en sintonía con la mayoría de artículos publicados (mejores supervivencia en el grupo de cirugía más RT (65\%), artículo de Dulguerov $\left.{ }^{18}\right)$. En el caso que se utilizó la QMT previa a la cirugía porque la enfermedad estaba localmente muy avanzada, es una opción a considerar, siendo utilizada de esta manera por otros autores como Resto $^{51}$ (en 3 de sus 27 pacientes), Loy ${ }^{37}$ (en los pacientes con grado C de Kadish utiliza QMT y RT preoperatoria) y Díaz $^{17}$ (en 5 de los 30 pacientes presentados, 4 eran estadío C), entre otros.

El tratamiento de exéresis tumoral mediante endoscopia transnasal es una opción cada vez más utilizada por más autores ${ }^{6,9,14,15,22,57,61,62}$. En nuestro caso donde se operó con endoscopia transnasal, el paciente presentaba un estadío B de Kadish y se consiguió una exéresis subtotal, pero con buena respuesta posterior a la RT y con un seguimiento de 8 años sin evidencia de enfermedad.

En el metaanálisis realizado por Devaiah ${ }^{14}$, para comparar cirugía abierta vs cirugía endoscópica, se presentan unos resultados donde el tratamiento endoscópico presenta mejor supervivencia siendo estadísticamente significativo $(\mathrm{p}=0,0019)$. Tras el análisis de ambos grupos (ver tabla 7), se observa que el grupo de cirugía abierta presenta mayor número de casos en estadío avanzado, con lo que los resultados deben ser tomados con mucha cautela.

En la mayoría de nuestros pacientes utilizamos la endoscopia para confirmar la exéresis completa cuando utilizamos el abordaje subcraneal.

El tratamiento con radioterapia postcirugía es la opción más adoptada por la mayoría de centros, y combinada con la cirugía, son las terapias que aportan mayor supervivencia y menor recidivas a los pacientes ${ }^{5,7,18,24}$.

Nuestros resultados en términos de supervivencia y periodo libre de enfermedad a los 5 años, si estimamos su valor según la curva de Kaplan Meier, son de 74,1\% y del $55,6 \%$ respectivamente. En los artículos de la literatura el
Tabla 7

Estadio Kadish modificado según tratamiento en el estudio de Devaiah

\begin{tabular}{|l|c|c|c|c|}
\hline \multirow{2}{*}{ Tipo tratamiento } & \multicolumn{5}{|c|}{ Estadío Kadish modificado } \\
\cline { 2 - 5 } & A & B & C & D (*) \\
\hline Cirugía abierta & 11 & 47 & 95 & 4 \\
\hline Endoscópica & 11 & 11 & 16 & 1 \\
\hline Asistida con endoscopio & 15 & 19 & 16 & 1 \\
\hline No cirugía & 3 & 6 & 33 & 2 \\
\hline
\end{tabular}

(*) Estadío D de Kadish: metástasis ganglionares cervicales o a distancia.

rango de supervivencia a los 5 años se sitúa entre el 50$90 \%$ y el periodo libre de enfermedad entre el $45-90 \% \%^{2,16}$, $17,37,41,42,48,60$. Cabe decir que nuestra población de pacientes se encuentran la mayoría en estadíos avanzados de la enfermedad, 2 estadíos B y 9 en estadíos C de Kadish, por lo que nuestros resultados los consideramos satisfactorios.

Por lo que respecta a las complicaciones de la cirugía, se produjeron 3 en el total de 11 casos, lo que representa un total del $27 \%$ en el total de nuestros pacientes. Si tomamos como referencia las series más largas de ENB, el rango varía de un 10 al 32\% $\%^{2,16,17,37,41,42,48,60}$, siendo algunas de ellas de gravedad, no siendo así en nuestra serie (dos osteomielitis crónica del colgajo que se curaron tras la retirada del colgajo y una fístula de LCR que se resolvió tras el sellado del defecto dural con fascia lata).

En los dos casos diagnosticados antes del año 2000 pero que fueron tratados durante el periodo de estudio, en un caso la recidiva se produjo a los 10 años de seguimiento y en el otro caso a los 7 años. Este hecho reafirma la opinión de muchos autores sobre que el seguimiento de este tipo de pacientes debe ser largo porque se producen recidivas después de mucho tiempo ${ }^{46}$.

El único factor pronóstico estadísticamente significativo que hemos podido extraer de nuestra población de pacientes ha sido el grado histológico de Hyams como predictor de menor periodo libre de enfermedad $(\mathrm{p}=0,02)$. Sobre la supervivencia el valor no era significativo $(\mathrm{p}=$ $0,1)$, debido seguramente al tamaño de la muestra, pero se observa una tendencia. Los grado III/IV tienen una supervivencia y un periodo libre de enfermedad a los 5 años estimado por la curva de Kaplan Meier de un $40 \%$ y un $20 \%$ respectivamente. En cambio los grado I/II tienen un $100 \%$ en los mismos parámetros. En la serie de Morita ${ }^{46}$, sobre 49 pacientes tratados en la Clínica Mayo en un periodo de 39 años, propone que el sistema de clasificación de Hyams es un método estadísticamente significativo mejor para predecir resultado comparado con el sistema de clasificación 
de Kadish. En el trabajo de Dulguerov ${ }^{18}$ se recogen varios artículos en los que comparan estos dos subgrupos de la clasificación de Hyams, siendo la media de supervivencia para el grupo de I/II de un 56\% y para el grupo III/IV de un $25 \%$, presentando una odds ratio de 6.18 (95\% IC 1.3029.3). El resto de factores pronósticos contemplados en otras series, como clasificación de Kadish, la existencia de metástasis ganglionares, la modalidad de tratamiento y la edad del diagnóstico, no han podido ser demostradas en nuestro estudio, en parte por el limitado número de pacientes incluidos.

\section{Conclusiones}

El ENB es un tumor con muy baja incidencia por lo que las series publicadas en la literatura incluyen pocos pacientes estudiados durante largos periodos de tiempo.

1. Nuestra opinión sobre el diagnóstico anatomopatológico del ENB es que la clasificación de Hyams es válida, incluyendo el grado IV como el más indiferenciado y de peor pronóstico. Aunque los criterios para considerar un tumor indiferenciado sean imprecisos, existe la posibilidad de que un grado IV presente una progresión hacia una mayor proliferación con células más indiferenciadas, por lo que puede perder el aspecto de ENB y etiquetarse erróneamente de tumor o carcinoma indiferenciado. Creemos que en este caso, el diagnostico de ENB debería prevalecer. No obstante, esta afirmación tal vez debería ser corroborada con la aplicación de la microscopía electrónica y, sobretodo, con nuevos estudios genéticos.

2. El protocolo de manejo terapéutico propuesto sería en primer lugar la cirugía como tratamiento de elección.

a. En los estadíos C de Kadish considerados operables, la resección craneofacial creemos que todavía debe ser la más utilizada, dado que hasta el momento actual no hay datos estadísticamente significativos para dar soporte a la endoscopia. En casos seleccionados, podría estar indicada la endoscopia transnasal siempre y cuando no haya invasión del parénquima cerebral.

b. En los estadíos B, individualizando el caso y según la experiencia de cada centro, podría estar justificada tanto la utilización de la resección craneofacial clásica como la cirugía endoscópica.

c. Para los estadíos A, localizados en las fosas nasales, consideramos que la cirugía debería ser endoscópica.

3. Por lo que respecta al tratamiento coadyuvante, la RT debería realizarse después de la cirugía en todos los casos, porque es lo que ha demostrado mayor supervivencia en las series.

4. La quimioterapia queda relegada a aquellos casos considerados irresecables, las recidivas o que presentan metástasis a distancia.

5. Bajo nuestro entender, el factor pronóstico más importante en el curso evolutivo del ENB sería la clasificación de Hyams, siendo el grado III y IV los que presentan menor periodo libre de enfermedad y probablemente menor supervivencia.

De todas formas, sería necesario un estudio probablemente multicéntrico incluyendo un mayor número de pacientes, siguiendo las mismas clasificaciones y comparando tipos de tratamiento para llegar a unas conclusiones sobre el diagnóstico, tratamiento y factores pronósticos con suficiente evidencia científica.

\section{Bibliografía}

1. Argiris, A., Dutra, J., Tseke, P., Haines, K.: Esthesioneuroblastoma: the Northwestern University experience. Laryngoscope 2003; 113: 155-60.

2. Bachar, G., Goldstein, D.P., Shah, M., Tandon, A., Ringash, J., Pond, G., Gullane, P.J., Perez-Ordonez, B., Gilbert, R.W., Brown, D.H., Gentili, F., O'Sullivan, B., Irish, J.C.: Esthesioneuroblastoma: The Princess Margaret Hospital experience. Head Neck 2008; 30: 1607-1614.

3. Berger, L., Luc, R., Richard, D.: L'esthesioneuroepithel iome olfactif. Bull Assoc Fr Etude Cancer 1924;13: 410-421.

4.Biller, H.F., Lawson, W., Sachdev, V.P., Som, P.: Esthesioneuroblastoma: surgical treatment without radiation. Laryngoscope 1990; 100: 1199-1201.

5. Bradley, P.J., Jones, N.S., Robertson, I.: Diagnosis and management of esthesioneuroblastoma. Curr Opin Otolaryngol Head Neck Surg 2003; 11: 112-118.

6. Bragg, T.M., Scianna, J., Kassam, A., Emami, B., Brown, H.G., Hacein-Bey, L., Clark, J.I., Muzaffar, K., Boulis, N., Prabhu, V.C.: Clinicopathological review: esthesioneuroblastoma.

7. Broich, G., Pagliari, A., Ottavani, F.: Esthesioneuroblastoma: a general review of the cases Publisher since the discovery of the tumor in 1924. Anticancer Res 1997; 17:2683-2706.

8. Butugan, O., Bogar, P., Sennes, Lu., Ognibene, R., Ishida, L., da Silveira, J.A., Rosemberg, S., Minita, A.: Olfactory neuroblatoma. A report of 5 cases. Rev Laryngol Otol Rhinol (Bord) 1993; 114: 359-363.

9. Castelnuovo, P., Bignami, M., Delù, G., Battaglia, P., Bignardi, M., Dallan: Endonasal endoscopic resection and radiotherapy in olfactory neuroblastoma: our experience. Head Neck 2007; 29: 845-850.

10. Chamberlain, M.C.: Treatment of intracranial metastatic esthesioneuroblastoma. Cancer 2002; 95: 243-248.

11. Chao, K.S., Kaplan, C., Simpson, J.R., Haughey, B., Spector, G.J., Sessions, D.G., Arquette, M.: Esthesioneuroblastoma: the impact of treatment modality. Head Neck 2001; 23: 749-757.

12. Cohen, Z.R., Marmor, E., Fuller, G.N., DeMonte, F.: 
Misdiagnosis of olfactory neuroblastoma. Neursurg Focus 2002; 12(5): e3.

13. Constantinidis, J., Steinhart, H., Koch, M., Buchfeider, M., Schaenzer, A., Wedenbecher, M., Iro, H.: Olfactory neuroblastoma; the University of Erlanger-Nuremberg experience 1975-2000. Otolaryngol Head Neck Surg 2004; 130:567-574.

14. Devaiah, A.K., Andreoli, M.T.: Treatment of Esthesioneuroblastoma: a 16-year meta-analysis of 361 patients. Laryngoscope 2009; 119: 1412-1416.

15. Devaiah, A.K., Larsen, C., Tawfik, O., O'Boynick, P., Hoover, L.A.: Esthesioneuroblastoma: endoscopic nasal and anterior craniotomy resection. Laryngoscope 2003; 113: 2086-2090.

16. Dias, F.L., Sa, G.M., Lima, R.A., Kligerman, J., Leoncio, M.P., Freitas, E.Q., Soares, J.R., Arcuri, R.A.: Patterns of failure and outcomes in esthesioneuroblastoma. Arch Otolaryngol Head and Neck Surg 2003; 129: 1186-1192.

17. Díaz, E.M. Jr, Johnigan, R.H. 3rd, Pero, C., El-Naggar, A.K., Roberts, D.B., Barrer, J.L., DeMonte, F.: Olfactory neuroblastoma: the 22-year experience at one comprehensive cancer center. Head Neck 2005; 27: 138-149.

18. Dulguerov, P., Allal, A.S., Calcaterra, T.C.: Esthesioneuroblastoma: a metaanalysis and review. Lancet Oncol 2001; 2: 683-690.

19. Dulgerov, P., Calcaterra, T.: Esthesioneuroblastoma: the UCLA experience 1970-1990. Laryngoscope 1992; 102:843-849.

20. Eriksen, J.G., Bastholt, L., Krogdahl, A.S., Hansen, O., Joergensen, K.E.: Esthesioneuroblastoma: what is the optimal treatment? Acta Oncol 2000; 39: 231-235.

21. Fliss, D.M., Spektor, S., Leider-Trejo, L., Abergel, A., Khafif, A., Amir, A., Gur, E., Cohen, J.T.: Skull base reconstruction after anterior subcranial tumor resection. Neursurg Focus 2002; 12(5): e10.

22. Folbe, A., Herzallah, I., Duvvuri, U., Bublik, M., Sargi, Z., Snyderman, C.H., Carrau, R., Casiano, R., Kassam, A.B., Morcos, J.J.: Endoscopic endonasal resection of esthesioneuroblastoma: a multicenter study. Am J Rhinol 2009; 23: 91-94.

23. Frierson, H.F. Jr, Ross, G.W., Mills, S.E., Frankfurter, A.: Olfactory neuroblastoma. Additional immunohistochemical characterization. Am J Clin Pathol 1990; 94: 547-553.

24. Gil-Carcedo, E., Gil-Carcedo, L.M., Vallejo, L.A., de Campos, J.M.: Esthesioneuroblastoma treatment. Acta Otorrinolaringol Esp 2005; 56: 389-395.

25. Hwank, S.K., Paek, S.H., Kim, D.G., Jeon, Y.K., Chi, J.G., Jung, H.W.: Olfactory neuroblastoma: survival rate and prognostic factor. J Neurooncol 2002; 59: 217-226.

26. Hyams, V.J.: Olfactory neuroblastoma. En Hyams VJ, Batsakis JG, Michaels L. Tumors of the upper respiratory tract and ear. Washington DC: Armed Forces Institute of Pathology 1988; 240-248.

27. Jekunen, A.P., Kairemo, K.J., Lehtonen, H.P., Kajanti, M.J.: Treatment of olfactory neuroblastoma. A report of 11 cases. Am J Clin Oncol 1996; 19: 375-378.

28. Jethanamest, D., Morris, L.G., Sikora, A.G., Kutler,
D.I.: Esthesioneuroblastoma: a population-based analysis of survival and prognostic factors. Arch Otolaryngol Head Neck Surg 2007; 133: 276-280.

29. Kadish, S., Goodman, M., Wang, C.C.: Olfactory neuroblastoma: a clinical analysis of 17 cases. Cancer 1976; 37: 1571-1576.

30. Kim, H.J., Kim, C.H., Lee, B.J., Cheng, Y.S., Kim, J.K., Choi, Y.S., Yoon, J.H.: Surgical treatment versus concurrent chemoradiotherapy as an inicial treatment modality in advanced olfactory neuroblastoma. Auris Nasus Larynx 2007; 34: 493-498.

31. Kim, J.W., Kong, I.G., Lee, C.H., Kim, D.Y., Rhee, C.S., Min, Y.G., Kim, C.W., Chung, J.H.: Expression of Bcl-2 in olfactory neuroblastoma and its association with chemotherapy and survival. Otolaryngol Head Neck Surg 2008: 139:708-712.

32. Kleinclaus, I., Floquet, J., Champigneulle, J., Perrin, C., Simon, C., Vignaud, J.M.: Olfactory neuroblastoma (esthesioneuroblastoma). A pathologic study of 7 cases. Ann Pathol 1993; 13: 241-246.

33. Koch, M., Constatinidis, J., Dimmier, A., Strauss, C., Iro, H.: Long-term experience in the therapy of esthesioneuroblastoma. Laryngorhinootologie 2006; 85) :723-730.

34. Lemoine, C., Serrano, E., Calvet, H., Delisie, M.B., Pessey, J.J.: Olfactory neurolbastoma. A propos of 12 cases. Rev Laryngol Otol Rhinol (Bord) 1992; 113: 185-189.

35. Levine, P.A., Gallagher, R., Cantrell, R.W.: Esthesioneuroblastoma: reflections of a 21 -year experience. Laryngoscope 1999; 109: 1539-1543.

36. Levine, P.A.: World Dr. Ogura approve of endoscopic resection of Esthesioneuroblastomas? An analysis of endoscopic resection data versus that of craniofacial resection. Laryngoscope 2009; 119: 3-7.

37. Loy, A.H., Reibel, J.G., Read, P.W., Thomas, C.Y., Newman, S.A., Jane, J.A., Levine, P.A.: Esthesioneuroblastoma: continued follow-up of a single institution's experience.

38. Lund, V.J., Howard, D., Wei, W., Spittle, M.: Olfactory neuroblastoma: past, present, and future? Laryngoscope 2003; 113: 502-507.

39. Martinez Subias, J., Domínguez Ugidos, L.J., Urpegui García, A., Sancho Serrano, E., Abenia Ingalaturre, J.M., Millán Guevara, J, Valles Varela, H.: Olfactory neuroblastoma. Review of seven cases. Acta Otorrinolaringol Esp 1998; 49:293-296.

40. Martel, J., Darrouzet, V., Duclos, J.Y., Bébéar, J.P., Stoll, D.: Olfactory neuroblastoma. Rev Laryngol Otol Rhinol (Bord) 2000; 121: 227-236.

41. McElroy, E.A. Jr, Buckner, J.C., Lewis, J.E.: Chemotherapy for advanced esthesioneuroblastoma: the Mayo Clinic experience. Neurosurg 1998; 42: 1023-1027.

42. McLean, J.N., Nunley, S.R., Klass, C., Moore, C., Müller, S., Johnstone, P.A.: Combined modality therapy of esthesioneuroblastoma. Otolaryngol Head Neck Surg 2007; 136: 998-1002.

43. Min, K.W.: Usefulness of electron microscopy in the diagnosis of "small" round cells tumors of the sinonasal region. Ultrastruct Pathol 1995; 19:,347-363. 
44. Mishima, Y., Nagasaki, E., Terui, Y., Irie, T., Takahashi, S., Ito, Y., Oguchi, M., Kawabata, K., Kamata, S., Hatake, K.: Combination chemotherapy (cyclophosphamide, doxorubicin and vincristina with continuos-infusion cisplatin and etoposide) and radiotherapy with ítem cell support can be beneficial for adolescents and adults with esthesioneuroblastoma. Cancer 2004; 101: 1437-1444.

45. Miyamoto, R.C., Gleich, L.L., Biddinger, P.W., Gluckman, J.L.: Esthesioneuroblastoma and sinonasal undifferenciated carcinoma: impact of histological grading and clinical staging on survival and prognosis. Laryngoscope 2000; 110:1262-1265.

46. Morita, A., Ebersold, M.J., Olsen, K.D., Foote, R.L., Lewis, J.E., Quast, L.M.: Esthesioneuroblastoma: prognosis and management. Neurosurgery 1993; 32: 706-714.

47. Nakao, K., Watanabe, K., Fujishiro, Y., Ebihara, Y., Asakage, T., Goto, A., Kawahara, N.: Olfactory neuroblastoma: long term clinical outcomes at a single institute between 1979 and 2003. Acta Otolaryungol Suppl 2007; (559): 113-117.

48. Nichols, A.C., Chan, A.W., Curry, W.T., Barrer, F.G., Deschler, D.G., Lin, D.T.: Esthesioneuroblastoma: the Massachussets eye and ear infirmary and Massachussets general hospital experience with craneofacial resection, proton beam radiation and chemotherapy: Skull base 2008; 18: 327-337.

49. Oskulaian, R.J. Jr., Jane, J.A. Sr, Dumont, A.S., Sheehan, J.M., Laurent, J.J., Levine, P.A.: Esthesioneuroblastoma: clinical presentation, radiological, and pathological features, treatment, review of the literatura, and the University of Virginia experience. Neursurg Focus 2002; 12 (5): e4.

50. Rastogi, M., Bhatt, M., Chufal, K., Srivastava, M., Pant, M., Srivastava, K., Mehrotra, S.: Esthesioneuroblastoma treated with non-craniofacial resection surgery followed by combined chemotherapy and radiosurgery: An alternative approach in limited sources. Jpn J Clin Oncol 2006; 36: 613-619.

51. Raveh, J., Turk, J.B., Lädrach, K., Seiler, R., Godoy, N., Chen, J., Paladino, J., Virag, M., Leibinger, K.: Extended anterior subcranial approach for skull base tumors: long-terms results. J Neurosurg 1995; 82: 1002-1010.

52. Resto, V.A., Eisele, D.W., Forastiere, A., Zahurak, M., Lee, D.J., Westra, W.H.: Esthesioneuroblastoma: the John Hopkins experience. Head Neck 2000; 22: 550-558.

53. Rinaldo, A., Ferlito, A., Shaha, A.R., Wei, W.I., Lund, V.J.: Esthesioneuroblastoma and cervical lymph node metastases: clinical and therapeutic implications. Actal Otolaryngol 2002; 122: 215-221.

54. Sakata, K., Aoki, Y., Karasawa, K., Nakagawa, K., Hasezawa, K., Muta, N., Terahara, A., Onogi, Y., Sasaki, Y., Akanuma, A., et al.: Esthesioneuroblastoma. A report of seven cases. Acta Oncol 1993; 32: 399-402.

55. Schrenzel, M.D., Higgins, R.J., Hinrichs, S.H., Smith, M.O., Torten, M.: Type C retroviral expression in spontaneous feline olfactory neuroblastomas. Acta Neuropathol 1990; 80:547-553.

56. Simon, J.H., Zhen, W., McCulloch, T.M., Hoffman, H.T., Paulino, A.C., Mayr, N.A., Buatti, J.M.: Esthesioneu- roblastoma: the University of Iowa experience 1978-1998. Laryngoscope 2001; 111: 488-493.

57. Sorensen, P.H., Wu, J.K., Berean, K.W., Lim, J.F., Donn, W., Frierson, H.F., Reynolds, C.P., López Terrada, D., Triche, T.J.: Olfactory neuroblastoma is a peripheral primitive neuroectodermal tumor related to Ewing Sarcoma. Proc Natl Acad Sci USA 1996; 93: 1038-1043.

58. Suriano, M., De Vicentiis, M., Colli, A., Benfari, G., Mascelli, A., Gallo, A.: Endoscopic treatment of esthesioneuroblastoma: a minimally invasive approach combined with radiation therapy. Otolaryngol Head Neck Surg 2007; 136:104-107.

59. Tandon, D.A., Bahadur, S., Mohanti, B.K., Rath, G.K.: Olfactory neuroblastoma: results of combined therapy. Indian J Cancer 1994; 31: 124-129.

60. Tatagiba, M., Samii, M., Dankoweit-Timpe, E., Aguiar, P.H., Osterwald, L., Babu, R., Ostertag, H.: Esthesioneuroblastoma with intracraneal extensión. Proliferative potencial and management. Arq Neuropsiquiatr 1995; 53: 557-586.

61. Theilgaard, S.A., Buchwald, C., Ingeholm, P., Kornum Larsen, S., Eriksen, J.G., Sand Hansen, H.: Esthesioneuroblastoma: a Danish demographic study of 40 patients registered between 1978 and 2000. Acta Otolaryngol 2003; 123:433-239.

62. Unger, F., Haselsberger, K., Walch, C., Stammberger, H., Papaefthymiou, G.: Combined endoscopic surgery and radiosurgery as treatment modality for olfactory neuroblastoma (esthesioneuroblastoma). Acta Neurochir 2005; 147: 595-601

63. Unger, F., Walch, C., Stammberger, H., Papaefthymiou, G., Haselberg, K., Pendl, G.: Olfactory neuroblastoma (esthesioneuroblastoma): report of six cases treated by a novel combination of endoscopic surgery and radiosurgery. Minim Invasive Neurosurg 2001; 44: 79-84.

64. Vergani, F., Pirola, E., Fiori, L., Pagni, F., Parmigiani, F., Sganzerla, E.P.: Combined transcranial and endoscopic nasal resection for esthesioneuroblastoma. Technical Note. J Neurosurg Sci 2007; 51: 99-102.

65. Walch, C., Stammberger, H., Unger, F., Anderhuber, W.: A new therapy concept in esthesioneuroblastoma. Laryngorhinolootologie 2000; 79: 743-748.

66. Wang, C.C., Chen, Y.L., Hsu, Y.S., Jung, S.M., Hao, S.P.: Transcranial resection of olfactory neuroblastoma. Skull base 2005 ; 15: 163-171.

67. Zafereo, M.E., Fakhri, S., Prayson, R., Batra, P.S., Lee, J., Lanza, D.C., Citardi, M.J.: Esthesioneuroblastoma: 25-year experience at a single institution. Otolaryngol Head Neck Surg 2008. 138: 452-458.

68. Zappia, J.J., Carroll, W.R., Wolf, G.T., Thornton, A.F., Ho, L., Krause, C.J.: Olfactory neuroblastoma: the results of modern treatment approaches at the University of Michigan. Head Neck 1993; 15: 190-196.

69. Zumegen, C., Michel, O.: Classification and prognosis of esthesioneuroblastoma based on 7 treated cases. Laryngorhinolootologie 2000; 79: 736-742. 
Muñoz, F.; Tresserras, P.; Montserrat, J.R.; Sancho, F.J.; Bartumeus, F.: Estudio retrospectivo de 11 casos de estesioneuroblastomas tratados en el Hospital Santa Creu i Sant Pau entre los años 2000 y 2008 más revisión de la literatura. Neurocirugía 2011; 22: 401-418.

\section{Comentarios al trabajo Estudio retrospectivo de 11 casos de estesioneuroblastomas tratados en el Hospital Santa Creu i Sant Pau entre los años 2000 y 2008 más revisión de la literatura De F. Muñoz y cols.}

Los autores estudian retrospectivamente una serie consecutiva de 11 casos de estesioneuroblastoma intervenidos durante un periodo de 8 años intentando definir las variables pronósticas más relevantes.

Los casos recogidos se estadiaron según las clasificaciones de Kadish y Dulgerov-Calcaterra predominando, a pesar de lo indolente de la sintomatología de debut, los estadíos avanzados (grados C de Kadish y T3, T4 de Dulgerov-Calcaterra).

El tratamiento de elección fue en la mayoría de los casos (8 de 11) la resección craneofacial mediante abordaje subcraneal con comprobación de la exéresis tumoral completa mediante endoscopia transnasal. La utilización de procedimientos endoscópicos estuvo condicionada por lo avanzado de la enfermedad, empleándose casi únicamente con finalidad diagnóstica y/o paliativa (biopsia más debulking). Todos los pacientes fueron sometidos a radioterapia y casi la mitad recibió quimioterapia aunque con distintos regímenes que incluyeron en todos los casos cisplatino y en distintas etapas de la enfermedad (antes de la cirugía, tras la cirugía o en el momento de la recidiva tumoral).

La incidencia de complicaciones fue relativamente baja (dos osteomielitis crónicas, una infección de la herida y una fístula de LCR) si consideramos la naturaleza infiltrativa de las lesiones, lo avanzado del proceso en la mayoría de los casos y la agresividad de los procedimientos terapéuticos aplicados.

El análisis de resultados (supervivencia y periodo libre de enfermedad) se realizó sobre nueve casos ya que dos se excluyeron por tratarse de recidivas de tumores tratados previamente y fuera del plazo escogido. El tiempo medio de seguimiento fue de 53,3 meses y un elevado número de pacientes ( 6 casos de 9 que participaron en el análisis de resultados) fue tratado en los cuatro últimos años del periodo de estudio seleccionado. Este hecho y el reducido tamaño muestral constituyen una limitación relevante para el análisis cuantitativo en una patología que presenta tanta heterogeneidad en su evolución.
La supervivencia a los dos años fue del $81,2 \%$ y durante el periodo de seguimiento sólo el $45,5 \%$ de los pacientes permanecía sin enfermedad. El resultado estuvo condicionado por la alta incidencia de casos en estadíos avanzados de la enfermedad, muchos de los cuales coincidían con histologías desfavorables (cinco casos con tumores indiferenciados: grados III y IV de Hyams) en los que la posibilidad de obtener un resultado satisfactorio se acepta que es sensiblemente inferior. El grado de diferenciación histológico fue la única variable asilada con valor pronóstico.

Los autores concluyen el trabajo con algunas recomendaciones terapéuticas entre las que se incluye limitar la indicación de cirugía endoscópica a los tumores muy localizados (estadíos A y algunos en estadío B), promover la administración de radioterapia postoperatoria puesto que proporciona un incremento significativo de la supervivencia mientras que la quimioterapia sólo parece desempeñar un papel secundario con fines paliativos y especialmente en los casos con metástasis a distancia.

El estesioneuroblastoma constituye un verdadero desafío terapéutico. En la mayoría de los casos y debido a lo indolente de la sintomatología inicial es diagnosticado cuando existe una invasión notable de las estructuras de vecindad. A pesar de ello y salvo en los casos en los que existan metástasis a distancia es posible plantear el tratamiento con fines curativos, por lo que parece indispensable disponer de equipos multidisciplinarios formado por cirujanos y oncólogos con experiencia suficiente y dominio de las diferentes alternativas terapéuticas (incluyendo la vía endoscópica) que trabajando de manera coordinada permitan la consecución del mejor resultado posible.

La propuesta de los autores de realizar un estudio multicéntrico en hospitales con volumen suficiente de casos, siguiendo criterios homogéneos de clasificación y adoptando pautas de tratamiento consensuadas es indispensable para la obtención de conclusiones válidas en una patología infrecuente y con gran variabilidad en su comportamiento.

J. Domínguez Tenerife 
Los autores han completado un detallado análisis retrospectivo de su experiencia en el manejo del estesioneuroblastoma, así como una exhaustiva revisión de la literatura al respecto. Entre las conclusiones del trabajo, destaca la discrepancia existente en torno a la categorización diagnostica del estesioneuroblastoma grado IV o indiferenciado ya que, precisamente, es el grado histológico el factor pronostico de mayor significación en la muestra analizada. Los autores deben ser felicitados por presentar resultados oncológicos satisfactorios con una aceptable morbilidad quirúrgica.

La resección craneofacial anterior clásica es el abordaje preferido por la mayoría de los centros involucrados en el tratamiento de tumores nasosinusales con extensión a la base craneal anterior. Sin embargo, en determinados centros de alta especialización en cirugía de base de cráneo, la resección craneofacial anterior vía exclusivamente endoscópica endonasal se ha impuesto como la técnica de elección para el tratamiento de este tipo de lesiones. El abordaje endoscópico endonasal a la fosa craneal anterior permite la resección completa de tumores con invasión de la base craneal, órbita, e incluso parénquima cerebral. Un aspecto no mencionado por los autores, pero de gran trascendencia, es la conveniencia de seccionar los bulbos olfatorios de manera rutinaria en grados B y C de Kadish, y confirmar intraoperatoriamente márgenes tumorales negativos a nivel nasosinusal, dural, de la periórbita, y de los tractos olfatorios. El limite lateral de resección de la vía endonasal endoscópica en la fosa craneal anterior se sitúa en la superficie superior de los nervios ópticos y en el meridiano de la órbita, ya que tanto el techo del canal óptico como la mitad medial del techo orbitario pueden ser abiertos vía endonasal, pero tumores con extensión más lateral serán abordados de manera más efectiva por la vía clásica.

Sin duda, es fundamental adquirir un conocimiento anatómico detallado, destreza con la técnica microquirúrgica bajo visualización endoscópica, y experiencia quirúrgica adecuada antes de aventurarse a la resección de tumores nasosinusales con invasión de la base craneal por vía exclusivamente endoscópica endonasal. Igualmente, el perfeccionamiento de las técnicas de reconstrucción mediante colgajo vascularizado nasoseptal o incluso mediante colgajo pericraneal (cuando el nasoseptal está infiltrado por tumor) es requisito imprescindible. Dicho esto, es necesario aclarar que la vía de abordaje o técnica empleada, ya sea clásica o endoscópica, no tiene la facultad de cambiar por si misma el grado histológico de la lesión, que es precisamente el factor pronóstico más importante. La belleza del abordaje endonasal estriba, no en su potencial para mejorar el pronostico lesional, sino en la consecución del mismo objetivo, la resección tumoral completa, sin necesidad de realizar craneotomía, retracción o manipulación cerebral, ni -en muchas ocasiones- incisiones externas, y por tanto con menor traumatismo quirúrgico y menor morbilidad asociada.

J.C. Fernández-Miranda Pittsburgh (PA), Estados Unidos

$* * * * * * * * * * * * * * * * * * * * * * * * * * * * *$

\section{Respuesta a los comentarios realizados}

Agradecemos encarecidamente los comentarios realizados sobre nuestro trabajo. Cabe decir que en el debate sobre la utilización de la resección craneofacial versus abordaje endoscópico endonasal la tendencia es a la sustitución del abordaje endoscópico por la vía clásica subcraneal. En nuestro centro utilizamos frecuentemente los abordajes endoscópicos para muy diversas patologías de cavidad nasal y base de cráneo en colaboración con los otorrinolaringólogos. Sin embargo, en los casos avanzados y con infiltración dural e incluso del parénquima cerebral consideramos la vía subcraneal clásica de Raveh como la más recomendable dado que requiere obtener márgenes de seguridad en patología tumoral maligna. En este abordaje transcraneal, la incisión es bicoronal por detrás del pelo, no se realiza ningún trépano frontal, sino que la craneotomía se realiza a través del seno frontal, con una sierra de $2 \mathrm{~mm}$ y se reconstruye con miniplacas, por lo que el resultado estético es totalmente satisfactorio. Otra ventaja significativa es que se consigue un gran colgajo de periostio vascularizado importantísimo para la reconstrucción dado que en muchas ocasiones no se dispone de un buen colgajo nasoseptal por infiltración tumoral. Dado que se extrae la raíz del hueso nasal, el ángulo de visión conseguida hace que la retracción cerebral prácticamente no exista. Además nosotros acompañamos el abordaje transcraneal combinado con el endoscopio endonasal para aprovechar las ventajas de las dos vías de abordaje.

A nuestro entender no hay por el momento superioridad del abordaje endoscópico endonasal sobre la resección craneofacial o abordaje subcraneal bien realizado, con unas ventajas de este último como son la obtención de un gran colgajo de periostio para la reconstrucción y una mayor visualización de toda la base craneal anterior sin los límites laterales que anteriormente se han descrito.

P. Tresserras Ribó Barcelona 